\title{
Transport at Interfaces in Lipid Membranes and Enantiomer Separation
}

O. Boyarkin ${ }^{1}$, S. Burger ${ }^{2}$, T. Franke ${ }^{2,4,5, *}$, T. Fraunholz ${ }^{1}$, R. H. W. Hoppe ${ }^{1,6}$, S. Kirschler ${ }^{2}$, K. Lindner ${ }^{2}$, M. A. Peter ${ }^{1,3, *}$, F. Strobl ${ }^{2,4}$, and A. Wixforth ${ }^{2,3,4}$

1 Institute of Mathematics, University of Augsburg, D-86159 Augsburg, Germany

2 Institute of Physics, University of Augsburg, D-86159 Augsburg, Germany

3 Augsburg Centre for Innovative Technologies, University of Augsburg, D-86159 Augsburg, Germany

4 Nanosystems Initiative Munich, Schellingstrasse 4, D-80799 München, Germany

5 Chair of Biomedical Engineering, School of Engineering, College of Science \& Engineering, Rankine Building, Oakfield Avenue, University of Glasgow, G12 8LT, UK

${ }^{6}$ Department of Mathematics, University of Houston, Houston, TX 77204-3008, USA

\begin{abstract}
We study the dynamics and formation of differently ordered lateral phases of interfacial lipid layers for two types of lipid systems, a vesicle-supported bilayer and a Langmuir-Blodgett monolayer, both in experiment and by simulation. Similarly, we investigate the dynamics of objects embedded in a simpler interface given by an air-water surface and demonstrate the surface-acousticwave-actuated separation of enantiomers (chiral objects) on the surface of the carrier fluid. It turns out that the dynamics and the separation of the phases do not only depend on parameters such as temperature, mobilities and line tension but also on the mechanics of the lipid layers subjected to exterior forces as, for instance, compression, extensional and shear forces in film-balance experiments. Since the mechanical behavior of lipid layers is viscoelastic, we use a modeling approach based on the incompressible NavierStokes equations with a viscoelastic stress term and a capillary term, a convective Jeffrey (Oldroyd) equation of viscoelasticity, and the Cahn-Hilliard equation with a transport term. The numerical simulations are based on $C^{0}$ interior-penalty discontinuous-Galerkin methods for the Cahn-Hilliard equation. Model-validation results and the verification of the simulation results by experimental data are presented. The feasibility of enantiomer separation by
\end{abstract}

${ }^{\star}$ Corresponding authors: T. Franke (thomas.franke@glasgow.ac.uk), M. A. Peter (malte.peter@math.uni-augsburg.de) 
surface-acoustic-wave-generated vorticity patterns is shown both experimentally and through numerical simulations. This technique is cost-effective and provides an extremely high time resolution of the dynamics of the separation process compared to more traditional approaches. The experimental setup is an enhanced Langmuir-Blodgett film balance with a surface-acoustic-wave generated vorticity pattern of the fluid, where model enantiomers (custommade photoresist particles) float on the surface of the carrier fluid. For the simulations, we propose a finite element immersed boundary method (FEIBM) for deformable enantiomers and a fictitious-domain approach based on a distributed Lagrangian multiplier finite element immersed boundary method (DLM-DEIBM) for rigid chiral objects, both of which lead to simulation results consistent with experiments.

\section{Introduction}

Biological cells are complex viscoelastic and soft objects which are composed of various components and compartments such as the plasma membrane, the nucleus, the endoplasmatic reticulum and a number of other cell organelles. All these compartments are surrounded by a bilayer membrane to separate the interior from its fluid environment and to regulate cell communication and maintain homeostasis. The biological concept of organelle-enclosing bilayers is a generic feature of all eukaryotic cells. However, on the molecular level these bilayers are far more heterogeneous and made of different amphiphilic molecules which form the "fluid scaffold" of the membrane. Therein, various types of macromolecules such as proteins and larger aggregates such as ion channels are embedded. This model was first described by Singer and Nicolson in 1972 [62] (cf. Figure 1) and has since then been refined and extended in several ways. One of those refinements is based on the observation that the bilayer membrane can undergo different phase-separation processes from a homogeneous membrane to a laterally structured heterogeneous membrane $[51,42]$. This decomposition is clearly a result of the multicomponent composition of the membrane from many types of lipids and a small amphiphilic molecule called cholesterol.

Lipids are one of the key building blocks of the membrane. They allow the cell to maintain morphological consistency and structural order in a mechanical as well a thermodynamic sense. Lipid membranes consist of two monolayers of lipid molecules, each molecule is made up of a polar hydrophilic end and a nonpolar hydrophobic tail. When exposed to water, the lipids tend to organize to form bilayers owing to the hydrophobic effect. Under appropriate conditions, the preferred shape of membranes is a closed shell, a so-called vesicle (cf. Figure 2). Vesicles can be created artificially in a relatively easy way, since lipids spontaneously self-assemble to form a closed bilayer structure and they have been analyzed thoroughly both theoretically and experimentally as model cell membranes. Such artificially created containers made of closed 


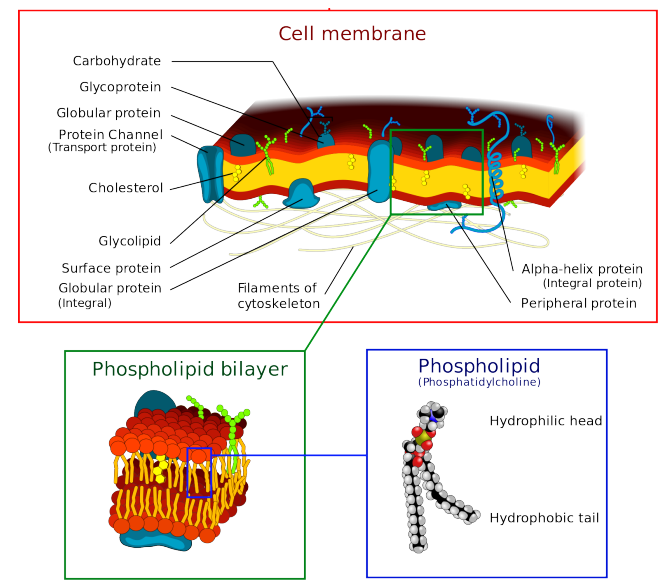

Fig. 1. Schematic sketch of a part of a cell membrane. (Adapted from: https://en.wikipedia.org/wiki/Cell_membrane)
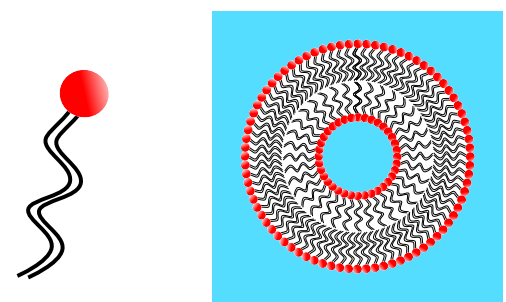

Fig. 2. Structure of a lipid (left), many of which form a closed shell, a so-called vesicle, under appropriate conditions (right). [29]

bilayers have also been used to deliver drugs, e.g. in liposomes. It is thus of vital importance to gain a profound understanding of the functioning of lipid membranes and how to manipulate them in order to comprehend their behavior and to ensure the designated effect of drugs. An important step towards this goal is to introduce mathematical models of the processes and to verify them by experimental observations. A key feature of the system is that the layers are essentially complex two-dimensional objects in a three-dimensional environment.

Biological lipid bilayers are mainly made up of phospholipids and behave like liquid crystals. The arrangement of the lipids can be changed depending on the situation (e.g., temperature) so that the membrane can exhibit several phases. The two liquid phases are distinctly ordered and are referred to as the liquid-ordered phase and the liquid-disordered phases. Because of the coexistence of phases, such a membrane can be thought of as a two-dimensional binary fluid. Below the characteristic temperature $T_{\mathrm{m}}$, which is a function of the concentrations of the liquid-ordered phase and the liquid-disordered 


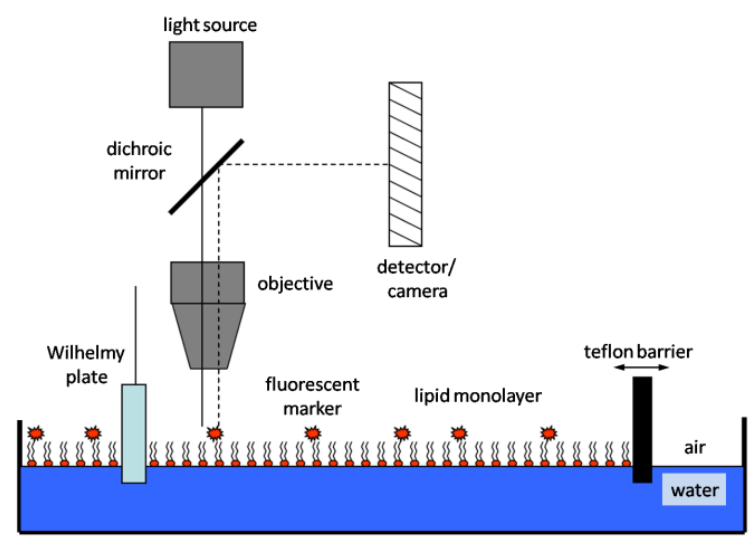

Fig. 3. Film balance experimental setup equipped with a microscopic observation unit. The total area of the lipid monolayer can be controlled by the motion of a teflon barrier.

phase, we can observe the phase-separation process. The kinetics of this separation process depend on whether the system is cooled to a metastable or an unstable region. In the metastable region, the decomposition of the phases is characterized by nucleation and growth. Cooling to the unstable region results in spinodal decomposition. Both types of separation have been observed experimentally.

Phase separation processes can be modeled by the Cahn-Hilliard equation [13]. In Section 2, as a first step, we compare qualitatively and quantitatively the results of experiments and simulations for temperature-induced lipid phase separation in artificially created vesicles neglecting mechanical effects. It is shown that the Cahn-Hilliard equation is an appropriate model in this context.

Since lipid layers often show a viscoelastic behavior, the Cahn-Hilliard equation has to be coupled with a viscoelastic fluid flow model such as a convective Jeffreys (Oldroyd) model (see [39, 44] for a detailed description). We are interested in experiments and numerical simulations where a lipid monolayer is spread onto the surface of a Langmuir-Blodgett trough filled with water. Phase separation is studied for a surface acoustic wave actuated fluid flow in the trough featuring four counter-rotating vortices at the upper surface of the trough (cf. Figure 3). In this case, the numerical simulation is based on a system of equations consisting of the incompressible Navier-Stokes equations with a viscoelastic stress term and a capillary term, a convective Jeffreys (Oldroyd) equation of viscoelasticity, and the Cahn-Hilliard equation with a transport term $[8,17,9]$. This system will be referred to as the NS/CJ/CH system. Both experimental measurements and numerical simulations for this system will be addressed in section 3 and section 4 , respectively. 

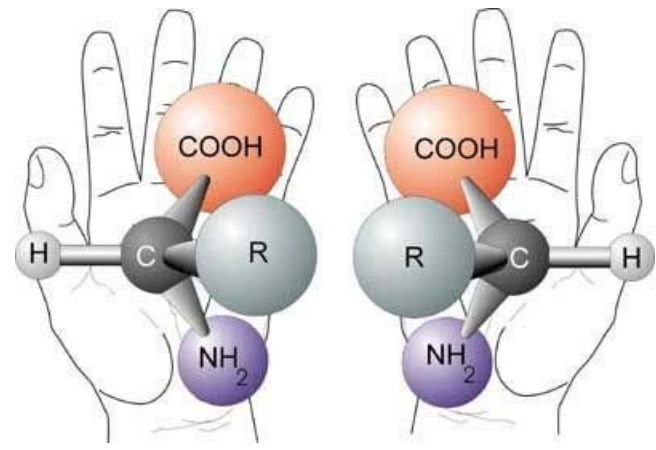

Fig. 4. Left- and right-handed enantiomers. (Source: https://en.wikipedia.org/wiki/Chirality_(chemistry))

Finally, we explore the experimental acoustic control and understanding of microflows that we gained in the previous sections to a practical problem of immense importance - the separation of enantiomers. Enantiomers are chiral geometric objects, where an object is said to be chiral if it is not identical to its mirror image. Since the word "chiral" stems from the Greek " $\chi \varepsilon \iota \rho$ ", which means "hand", one distinguishes enantiomers by their handedness (right- resp. left-handedness; cf. Figure 4).

In chemistry, chirality refers to a molecule, which is not superposable on its mirror image. Compounds consisting of molecules of the same handedness are called enantiopure or unichiral, whereas compounds consisting of the same amount of right- and left-handed enantiomers are referred to as racemic. Since the chemical synthesis of enantiomers usually gives rise to racemic compounds, enantiomer separation plays a significant role in agrochemical, electronic, and pharmaceutical as well as food, flavor and fragrance industries. Traditional separation technologies are based on gas or high pressure liquid chromatography, capillary electrophoresis, or nuclear magnetic resonance, but most of them are slow and require costly chiral media.

A different approach uses the fact that enantiomers drift in microflows with a direction depending on their chirality [46, 48]. Enantiomer separation using a quadrupolar force field generated by surface acoustic waves has been theoretically predicted in [43] for simple idealized chiral objects.

In section 5, we report both on an experimental setup for surface-acousticwave-actuated enantiomer separation and the mathematical modeling and numerical simulation of this setup by the finite element immersed boundary method and the distributed Lagrangian multiplier finite element immersed boundary method. The results confirm that the setup is capable of separating enantiomers by their handedness. 


\section{Phase separation and decomposition in a lipid bilayer membrane}

This section deals with the phase-separation process of a bilayer system, which is caused by lateral demixing of its multiple molecular components into different domains and describes the demixing in the framework of a Cahn-Hilliard model.

Domain structures can occur on length scales, which are amenable to optical light microscopy (several micrometers), though it is assumed that they also develop at scales of $10-100 \mathrm{~nm}$ and have been associated within the so called "lipid raft hypothesis" $[61,36]$ in biological cells. This hypothesis links the formation of a phase-separated membrane and its structure to biological functions, e.g. to control adhesion and cell signaling. It is hypothesized that the decomposition of the phase is directly related to molecular organization of proteins and other macromolecules, e.g. for adhesion, and is essential for physiological and cellular processes.

Here, to reduce the cell membrane complexity, we use Giant Unilamellar Vesicles (GUVs) as a simple model system, which is also accessible to light microscopy. GUVs are composed of a well-controlled number of different lipid types and cholesterol but still display the basic properties of cell membranes. Over the past years, there has been extensive experimental effort undertaken to study ternary lipid systems $[2,4,68,70]$ consisting of two types of phosphatidylcholines (PC) such as 1,2-Dipalmitoyl-sn-glycero-3-phosphocholine (DPPC) and 1,2-Dioleoyl-sn-glycero-3-phosphocholine (DOPC) and cholesterol as the third component. The phase separation of this system has been found to occur in two different ways, either by a nucleation process with subsequent ripening of the discrete domains into larger domains or by a spinodal decomposition process [69].

From a theoretical perspective, there have been a number of studies to reproduce the experimental findings qualitatively. However, quantitative comparison of the theoretical simulations with experimental data is rare [42]. Here, we address this shortcoming by directly comparing the temporal development of liquid-ordered domains in a GUV bilayer system. We quantitatively relate the optically observed domains in our experiments with a numerical simulation based on the Cahn-Hilliard equation.

\subsection{Vesicle experiments}

We produce GUVs with diameters of up to $200 \mu \mathrm{m}$ using the electroformation method as described by Angelova et al. [1]. In short terms, a small volume $(5 \mu \mathrm{l})$ of a $1 \mathrm{mg} / \mathrm{ml}$ total lipid in chloroform solution is deposited onto an ITO coated microscope slide and the solvent is evaporated. A second ITO slide is positioned opposite to the slide with the dried lipid spot and the gap between two slides is filled with distilled water or an aqueous solution containing sucrose. Then, an electric field of $10 \mathrm{~Hz}$ and $10 \mathrm{~V} / \mathrm{cm}$ is applied and kept 
constant overnight (at least 6 hours) and the GUVs form during this period. In all experiments, we use a mixture of $60 \mathrm{~mol} \%$ lipid and $40 \mathrm{~mol} \%$ cholesterol. Among the lipid part, we use the ratio of DOPC:DPPC:DPPG (5:4:1). Note that in addition to the commonly used DOPC/DPPC system, we added small traces of negatively charged 1,2-Dipalmitoyl-sn-glycero-3-phospho-rac(1-glycerol) (DPPG). In previous experiments, we found that using DPPG enables supercooling of the vesicles and prevents undesired and uncontrolled spontaneous prematuring of the vesicles. The lipids and cholesterol $(>99 \%)$ were purchased from Sigma Aldrich (Munich, Germany) and were used without further purification. The visualization of domains was enhanced by the fluorescence marker Texas Red (TR) which was also obtained from Sigma Aldrich. For observations, we used a Zeiss Axiovert 200 inverted fluorescence microscope (Zeiss, Oberkochen, Germany) and a CCD camera (Hamamatsu, Herrsching, Germany) for video recording. All experiments were performed in a custom-made and temperature-controlled fluidic chamber and observations were directly done after GUV formation. Therefore, the GUVs were supercooled below the critical phase-transition temperature $T_{m}$ and then illuminated with the microscope lamp to observe the spontaneous phase decomposition of the bilayer membrane. A typical temporal sequence of fluorescence micrograph frames is depicted in the right column of Figure 5.

In the first micrograph, a continuous and still diffuse pattern of domains can be seen, which closely resembles what is expected in the regime of spinodal decomposition. In the second frame, discrete yet connected domains have been already formed, which are, however, still far away from their circular equilibrium shape. In the further course of the experiment (micrograph 3), the domains quickly relax into a circular shape and start to grow mainly by coalescing with neighboring domains [12].

\subsection{Cahn-Hilliard simulations}

The challenge for the numerical simulation is to reproduce both the qualitative topological features as well as the quantitative temporal development and the domain size correctly. To model bilayers, a number of different methods have been applied including Monte-Carlo methods [38] or molecular-dynamics simulations [3]. In the past, phase-field models have been successfully used to reproduce complex microstructures [14]. Here, we use a Cahn-Hilliard model, which has been applied to a number of diverse problems before $[66,6]$ and which is discussed elsewhere in more detail [23, 52].

We capture the temporal decomposition into two different phases by introducing the mole fraction $c$ as an order parameter of the system. The values $c=1$ and $c=0$ indicate the pure phases and are represented in white and black, respectively, in the simulation pattern in Figure 5 (left column). We determine the order parameter $c$ from an equation of Cahn-Hilliard type

$$
\frac{\partial c}{\partial t}-\nabla \cdot\left[M \nabla\left(f^{\prime}(c)-\epsilon^{2} \Delta c\right)\right]=0 .
$$




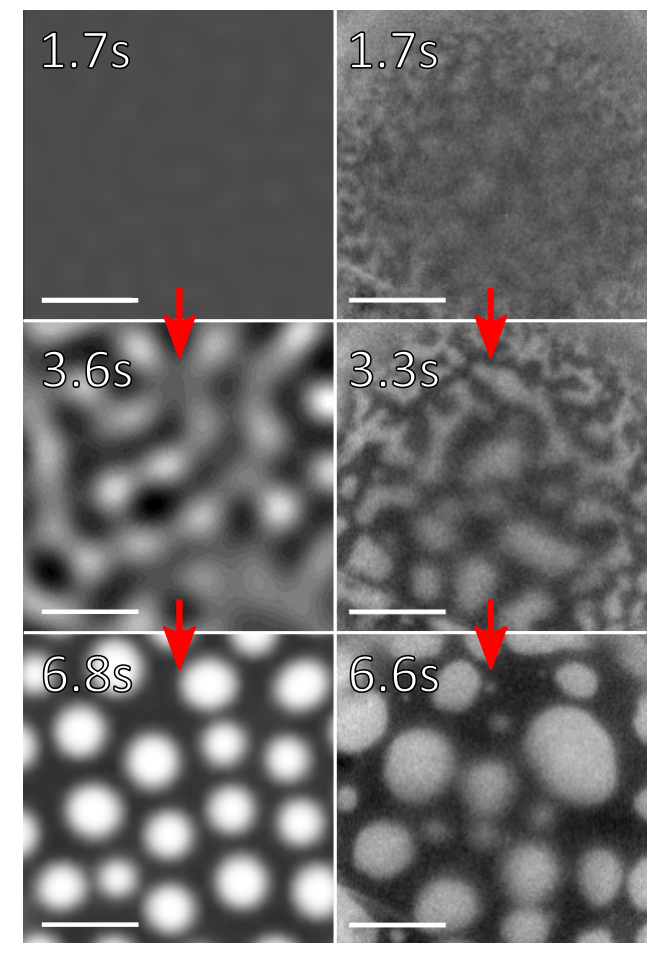

Fig. 5. Comparison of the temporal evolution of domain patterns obtained from a numerical simulation (left) and in the corresponding experiment (right micrographs). Each set of images shows the same bilayer changing its domain morphology during the phase decomposition. The numbers in the images indicate the time that has passed since the onset of phase decomposition. The time difference between the images is chosen such that each image shows a representative pattern that is observed in the distinct phases of decomposition illustrated in Figure 6. The scalebar is $10 \mu \mathrm{m}$. Reprinted with permission from S. Burger et al., Comparative study of the dynamics of lipid membrane phase decomposition in experiment and simulation. Langmuir $\mathbf{2 9}$ (25), 7565-7570. Copyright 2013 American Chemical Society.

Here, $f=f(c)$ is the local free energy per unit of area. In the first approach, we use a double-well potential $f(c):=\varphi c^{2}(c-1)^{2}$ with a scaling parameter $\varphi$. The parameter $M$ is the mobility which depends on the diffusion constants $D_{0}$ and $D_{1}$ of both phases according to $M=D_{0}(1-c)+D_{1} c$. For simplicity, we assume the same diffusion coefficients for both phases described by an effective constant $D$ [54], i.e. $M=D$. The parameter $\epsilon$ describes the energy cost of the boundaries of the two phases by the energy term $\frac{\epsilon^{2}}{2}|\nabla c|^{2}$. In this model, the physical properties of the system are captured by the three control parameters $D\left[\frac{m^{2}}{s}\right], \varphi\left[\frac{J}{m^{2}}\right]$, and $\epsilon^{2}[J]$. We explore the solution of the CahnHilliard equation and the temporal development of the phases and their do- 
main size systematically on these control parameters and compare them with the experiments. The simulations were performed using a $C^{0}$-interior-penalty discontinuous-Galerkin finite-element method [71, 28], which has proved to be stable and efficient for such kind of problems. This method was particularly chosen to account for the fourth-order spatial derivatives of the Cahn-Hilliard equation. In this way, it was not necessary to introduce a coupled system of partial differential equations and instead we could use the standard secondorder Lagrangian finite elements. We solve the nonlinear discrete equation with periodic boundary conditions by a Newton method. The triangulation was carefully chosen to avoid artificial symmetry effects, which could have been introduced by the discretization otherwise.

\subsection{Comparison of Cahn-Hilliard simulations and experiments}

We match the simulation to the microscopic observation and find optimal values for the control parameters $D\left[\frac{m^{2}}{s}\right], \varphi\left[\frac{J}{m^{2}}\right]$ and $\epsilon^{2}[J]$ by comparing the temporal development of mean domain sizes in both experiment and simulation. Since the domain size is difficult to determine directly from a distribution of sizes we analyze the micrographs and simulation plots with a 2D Fast Fourier Transform (FFT) and use the structure factor of a given mode $q$ to determine the mean domain size $1 /\langle q\rangle$ from

$$
\langle q\rangle:=\frac{\mathcal{S}(q) q}{\sum_{q} \mathcal{S}(q)}
$$

The structure factor is obtained from the image grey value $a\left(p_{j}\right)$ at $p_{j}$ depending on position $p_{j}$ and the scaling factor $N$ given by the number of $q_{j}$. For comparison, we plot the mean domain size against time as shown in Figure 6 (upper-left image) for parameter values of $D=1.0 \mu \mathrm{m}^{2} / \mathrm{s}, \varphi=4.0$ $\mathrm{J} / \mathrm{m}^{2}$ and $\epsilon^{2}=1.0 \cdot 10^{-12} \mathrm{~J}$ together with the experimental domain size. At first, it is apparent that the domain growth occurs in three distinct temporal periods in the simulations as well as in the experiments. It can be characterized by a slow initial domain growth, followed by accelerated growth and then, finally, a reduced growth approaching the final domain size. In the first period which ends at $t \approx 2$ seconds for the simulations and at $t \approx 1.5$ seconds for the experiments, we observe the spinodal decomposition of the membrane, which is also depicted in the upper panel of Figure 5. The second period lasts about 3 seconds in the experiment and about 2.5 seconds in the simulation and is characterized by an enhanced domain growth rate of a factor of 8 . Domain growth in this period is dominated by coalescence of adjacent small domains in the experiments while, in the simulations, domain formation still occurs as depicted in the middle panel of Figure 5 . In the last period, which sets in at $t \approx$ $4.5 \mathrm{~s}$ (the lower panel of Figure 5), matured and circular equilibrium domain shapes have been formed with a significant decrease in growth rate. The mean diameter at the end of the considered interval is approximately $5.0 \mu \mathrm{m}$ for both 
simulations and experiments. These qualitative and quantitative agreements of our simulations with the experimental data confirm that we capture the basic physics of the decomposition.

However, there are also quantitative differences, which we address in the following systematic parameter study as shown in the plots of Figure 6 . Therefore, we keep two of the control parameters constant and vary the remaining parameter. The sensitivity of the domain growth on the diffusion parameter $D$ is plotted in the top right image of Figure 6. As qualitatively expected, the domain growth rate increases with increasing values of $D$ because lipids are more mobile and can achieve the energetically favorable states faster. The simulation parameter $D$ is related to the physical lateral diffusion coefficient in lipid bilayers and has been measured to be of the order of $1-20 \mu \mathrm{m}^{2} / \mathrm{s}$ using various types of methods and lipid systems $[20,26,54,40]$. The values for the ternary system containing the lipids DOPC, DPPC and cholesterol have been given by Orädd [54] and were used to guide this parameter study. The other two control parameters $\varphi$ and $\epsilon^{2}$ are addressed in the lower-right and -left image of Figure 6, respectively. It is important to point out that unlike $D$, these two parameters cannot directly be correlated to physically measured values from the literature. Although, for example, the parameter $\epsilon^{2}$ controls the energy associated with the boundary of the two domains, it cannot be identified with the line tension [57]. To relate $\varphi$ and $\epsilon^{2}$ directly to physically-measured quantities, another model would be necessary, which clearly exceeds the frame of this study but is interesting to consider in the future. In the lower-left image, the dependence on $\varphi$ indicates that for larger $\varphi$ the decomposition occurs earlier and the rate of growth is enhanced. Since the parameter $\varphi$ characterizes the shape of the double-well potential and increases its steepness, energetically unfavorable states relax more rapidly into the equilibrium states. The last control parameter $\epsilon^{2}$ plotted in the right image strongly affects the final size of the domains. Together with the experimentally measured value, it was therefore taken to guide the simulations.

However, despite the good agreement with the experiments, some features of the domain growth still remain challenging. First, as can be seen from Figure 6 , the initial values of domain sizes do not correspond well. This cannot be compensated by variation of the control parameters because, as becomes clear from Figure 6, the initial domain size only weakly depends on the chosen values of $D, \varphi$ and $\epsilon^{2}$. We hypothesize that the larger value for the size in the experiment is attributed to incomplete mixing in the beginning and may also be affected by the limited resolution of the optical system as well as nonlinear partitioning of the Texas Red marker between the two phases. Another characteristic difference to the experiments is that, in the second domain growth period, the experiment is mainly driven by coalescence while, in the simulations, coalescence occurs as well but seems to be less dominant. We can speculate that one reason for this behavior is the role of the traces of negatively charged DPPG used in the experiments. Moreover, our model does not account for the mechanical effects in the system and the mechanical 

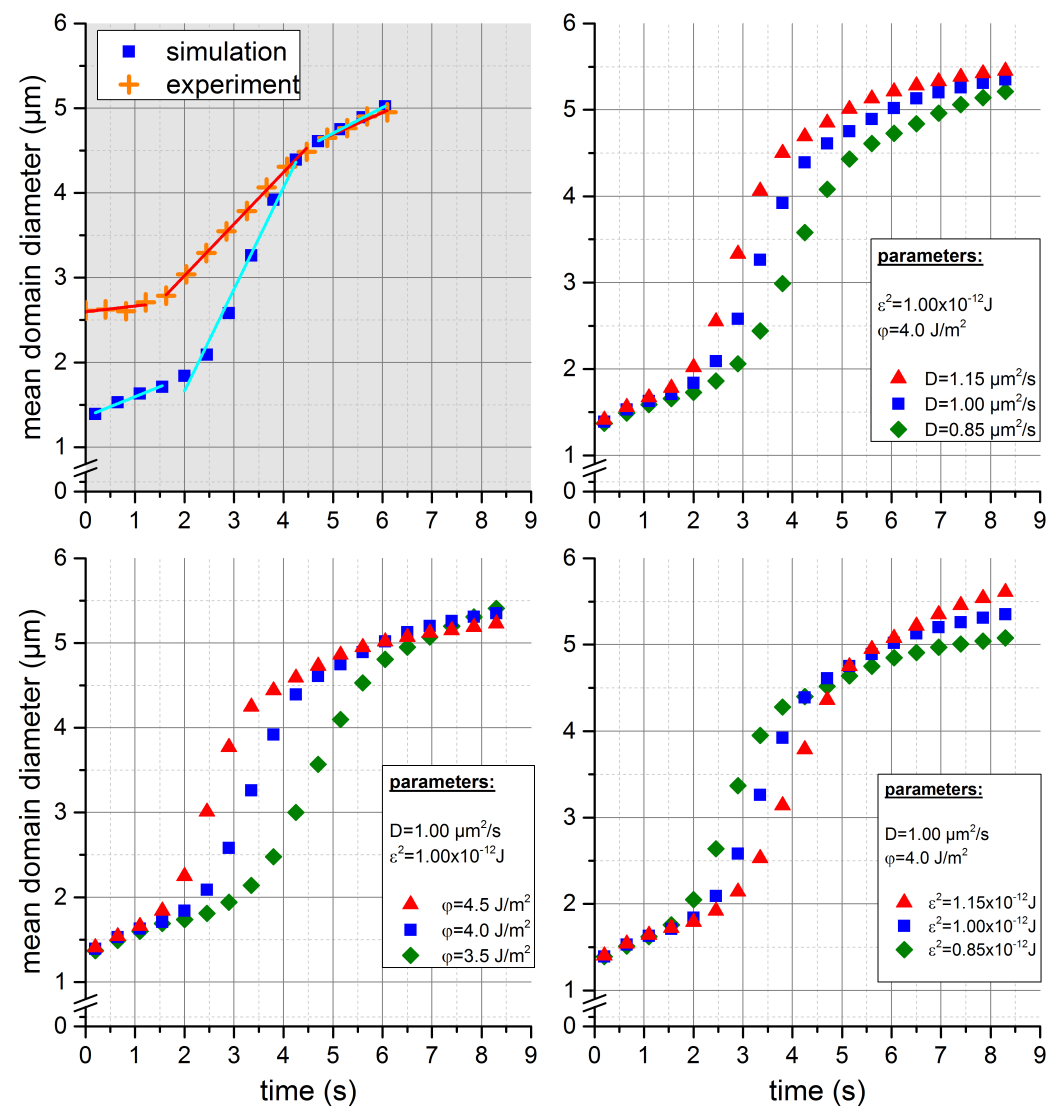

Fig. 6. Top Left: Evolution of the mean domain diameter during the phase transition. Orange crosses mark experimental data while blue squares represent results of numerical simulations $\left(D=1 \frac{\mu \mathrm{m}^{2}}{\mathrm{~s}}, \epsilon^{2}=1 \cdot 10^{-12} \mathrm{~J}, \varphi=4 \frac{\mathrm{J}}{\mathrm{m}^{2}}\right)$. Top Right: Results of numerical simulations with varying mobility parameter $D$. Bottom Left: Results of numerical simulations with varying local free energy scaling parameter $\varphi$. Bottom Right: Results of numerical simulations with varying effective line tension parameter $\epsilon^{2}$. Note that the curve represented by blue squares is identical in all panels. Reprinted with permission from S. Burger et al., Comparative study of the dynamics of lipid membrane phase decomposition in experiment and simulation. Langmuir 29 (25), 7565-7570. Copyright 2013 American Chemical Society.

stress that certainly impact the values of the control parameters. We address the dynamics of a lipid system in the subsequent section by compressing a lipid monolayer on a Langmuir film balance and address the dynamic effects that include viscoelastic shear properties. 

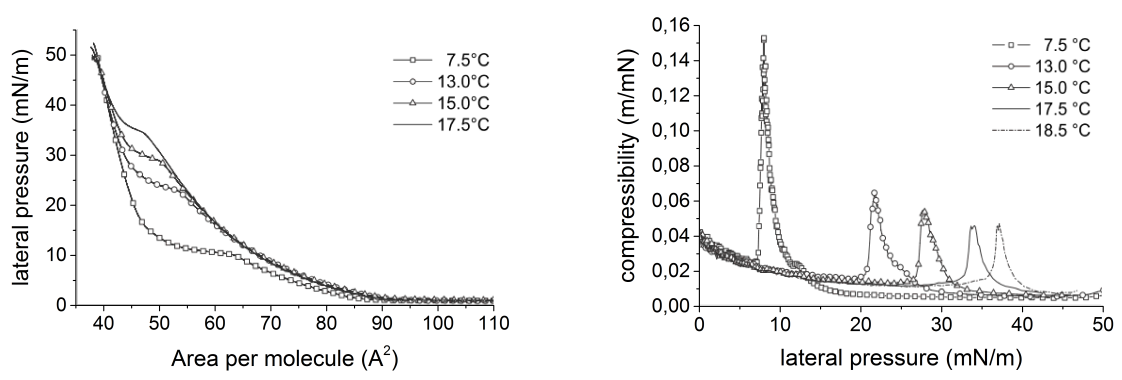

Fig. 7. (adapted from [63]) a) Typical isotherms for DPPC monolayers at different temperatures; b) The area elasticity of the monolayer approaches a maximum at the phase transition.

\section{Probing mechanics of lipid monolayers by acoustic actuation}

In the previous section, we focused on the decomposition of a lipid bilayer system. One of the limitations was that mechanical effects were not taken into account. Here, we use a slightly different system, a lipid monolayer, spread on a Langmuir trough film balance, and investigate the viscoelastic response to an external mechanical excitation.

There has already been a vast amount of work dedicated to investigate lipid monolayers and bilayers [55] (see also the book [51] for a better understanding of the physical properties of these systems). For monolayers, mechanical properties such as the area elasticity and dilatational viscosity have been studied intensively depending on the thermodynamic state of lipid monolayers. Most natural lipids typically show a first-order transition between a liquid-expanded (LE) and a liquid-condensed (LC) phase. In monolayers, this transition becomes apparent in a pronounced plateau in the surface pressure vs. molecular area isotherm, which is commonly recorded on a Langmuir film balance, see Figure 7.

In a Langmuir film balance, the lateral pressure is measured as a function of the molecular area and is given as the difference in surface pressure in the presence and the absence of the lipid monolayer. Thermodynamically, the lateral pressure is the derivative of the surface free energy with respect to the area per molecule. The isothermal compressibility and the isobaric thermal expansivity as material parameters are then given as the partial derivatives of the molecular area with respect to the pressure and temperature, respectively. The horizontal section in Figure 7(a) suggests that the transition is discontinuous and of first order. In the vicinity of a critical state (near the phase transition) the thermodynamic fluctuations of a system approach a maximum [63]. 
Since these fluctuations are closely related to the susceptibilities and hence linked to the material properties, parameters like area elasticity, heat capacity or electrical conductivity change dramatically and often take extreme values in the transition region. Whereas these phenomena have been investigated intensively for the area properties, much less work has been done to investigate the shear properties of these systems. Here, we focus on the shear properties of lipid monolayers in the vicinity of their LE-LC transition. The LC-regime has been investigated extensively due to its outstanding relevance for the physics of breathing and due to its good accessibility for established rheological methods [41, 24, 15]. In contrast, lipid layers in the liquid-expanded regime do not show any shear elasticity and have very low shear viscosities. The ratio of drag forces arising from the surface to those arising from the subphase underneath is usually described by the Boussinesq number

$$
\mathrm{Bo}=\frac{\text { surface drag }}{\text { subface drag }}=\frac{\eta_{s}}{\eta_{0}} \cdot \frac{R}{A},
$$

where $\eta_{s}$ and $\eta_{0}$ are the viscosities of inter- and subphase, respectively, and the ratio $R / A$ describes the geometry of the probe used, i.e. the ratio of contact perimeter with the interphase and contact area with the subphase [10]. For direct shear measurements, where $R / A$ is limited by technical means, low surface viscosities are difficult to measure. While some work has been done for LE-phase of lipid monolayers at constant surface pressure [60], very little data is available for the main transition regime [58, 56, 21, 59]. Especially the influence of the monolayer microstructure on the shear properties has not been investigated systematically, even though it is well known that the structure of LC-domains in the phase-transition regime is sensitive to their growth velocity and impurities as for example tracer particles or fluorophore molecules [49, 50, 33].

To probe the monolayers mechanically, we introduce here a new method for investigating the shear viscosity and elasticity of lipid monolayers as functions of surface pressure. We show that both viscous and elastic properties depend on the total area of LC-domains as well as on their microstructure.

For all experiments, a customized Langmuir trough from NIMA was used. The surface area can be adjusted by a movable teflon barrier. The surface pressure is measured using a Wilhelmy plate and a NIMA force sensor. A focused interdigital acoustic transducer was installed at the bottom of the trough for the excitation of an acoustic flow in the observed region as shown in Figure 3 and Figure 24. The chip is powered by a high frequency generator and an amplifier at its resonance frequency with an input amplitude of $9 \mathrm{dBm}$. The trough is covered by a plastic lid to protect the interface from potential air motion. Additionally, several teflon barriers spanning the full height of the trough were introduced to suppress large scale streaming (see Figure 8). The temperature of the teflon trough and the subphase was stabilized by a heating bath at $23^{\circ} \mathrm{C}$. The monolayer was observed through a glass window with an upright fluorescent microscope equipped with a Photron FastCam camera. 


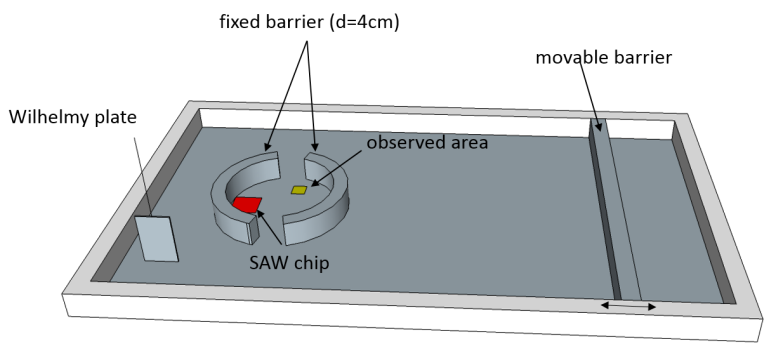

Fig. 8. Top view of the trough geometry. Two additional semicircular barriers decouple the observed area from large scale flow excited by external disturbance. The SAW chip (red) is mounted at the bottom such that the fluid streamline with maximal velocity crosses the observed monolayer area.

Dipalmitoyl-sn-glycero-phosphatidylcholine (DPPC) from Avanti Polar Lipids (Alabaster, USA) and Texas Red-DHPE from Invitrogen were used without further purification. All other chemicals were purchased from Sigma Aldrich (Germany). Ultrapure water ( $>18 \mathrm{M} \Omega \mathrm{cm}$, PureAqua, Tuttlingen, Germany) was used for all aqueous solutions. A solution of 10 M HEPES buffer and $90 \mathrm{mM} \mathrm{NaCl}$ was adjusted to a $\mathrm{pH}$ of 7.0 and was used as subphase for all experiments and shields electrostatic interactions. DPPC and Texas-Red-DHPE with a molar ratio of $0.5 \%$ were dissolved in chloroform with an overall concentration of $1 \mathrm{mg} / \mathrm{ml}$. Lipid monolayers were formed by slowly pipetting a fixed amount of lipid solution onto the fluid surface. Before starting the experiments, the monolayer was equilibrated for ten minutes to allow for the evaporation of chloroform traces.

After equilibration, the monolayer was compressed slowly until first LC domains appear. From here, the pressure was increased in steps of $0.5 \mathrm{mN} / \mathrm{m}$ until the domains were strongly compressed. Afterwards the barrier motion was reversed and the area was increased again in steps of $0.5 \mathrm{mN} / \mathrm{m}$. At each step, the monolayer was equilibrated at constant area for five minutes before the SAW flow was repeatedly turned on and off for time intervals of $5 \mathrm{~s}$. To track the flow, videos of the domain motion were captured with a frame rate of 125 fps.

Captured videos were first analyzed by a script based on the open source PIVlab toolkit22 to obtain the velocity field for the complete field of view at different time steps. 20 frames were used for the particle imaging velocimetry (PIV) analysis of one time step to reduce motion and data processing artefacts. Hence, the velocity information for further processing has a temporal 

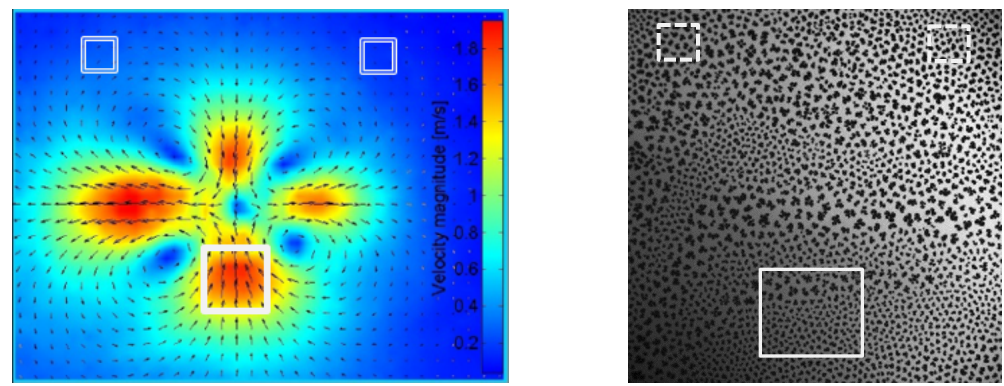

Fig. 9. Typical flow profile obtained from the PIV analysis. The larger solid frame indicates the region of interest, the double lined squares indicate the reference fields.

resolution of $160 \mathrm{~ms}$. A region of interest of $50 \times 50$ pixels in one center stream line of the quadrupolar flow field was chosen and the $y$-component of the velocity field was averaged spatially. We refer to the velocity obtained in this way as $v_{\mathrm{y}}$ in the following. In the same way, a reference velocity $v_{\text {ref,y }}$ was determined by spatially averaging the flow fields in the two opposing corners of the field of view.

The structure of the (dark) LC-domains within the region of interest was analyzed by using ImageJ-scripts. The area ratio $\alpha=\frac{A_{\mathrm{LC}}}{A_{\mathrm{LE}}+A_{\mathrm{LC}}}$ was determined by "thresholding" and the connectivity $\zeta$ by the BoneJ plugin from ImageJ. The latter value is derived from the Euler characteristic of the indexed picture and measures the "porosity" of the LC-domains (e.g. small, isolated domains will result in very negative $\zeta$ values).

\subsection{Experimental results and discussion}

\section{Velocity amplitude and viscosity}

In a first set of experiments, we periodically excite acoustic streaming by repeatedly switching the SAW on and off for $5 \mathrm{~s}$ at a period of $10 \mathrm{~s}$. As a response to this excitation the differential velocity $\Delta v_{\mathrm{y}}=v_{\mathrm{y}}-v_{\text {ref,y }}$ in the selected ROI has been analyzed for different thermodynamic states of the membrane. Figure 10 gives an overview of the measured data. We introduced the differential velocity $\triangle v_{\mathrm{y}}$ to compensate vibration artefacts in the experimental setup from various sources as small vibration of the damping table or air flow. Since usually $\Delta v_{\mathrm{y}} \gg v_{\text {ref,y }}$, the error introduced here is very small.

One can clearly see from Figure 10 that the maximum amplitude of $\Delta v_{\mathrm{y}}$ decreases with higher surface pressure as can be expected as consequence of increasing surface viscosity at higher surface pressures. For quantification, we derive $v_{\mathrm{y} \text {,max }}$ by averaging over ten data points from each period just before the SAW is switched off. When we increase the surface area after compression into the LE-regime, we observe very strong hysteresis effects and there is no clear correlation to the measured surface pressure (see Figure 11a). Hysteresis 

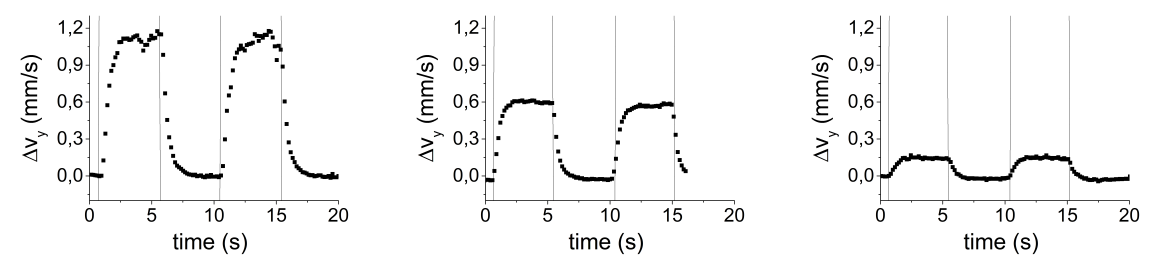

Fig. 10. $\triangle v_{\mathrm{y}}$ as a function of time for different membrane states (ratio of the LC phase), i.e. different area ratios. a) $\alpha=50 \%$ b) $\alpha=60 \%$ c) $\alpha=80 \%$. With rising LCratio, the velocity amplitude decreases significantly as a result of increasing viscosity. The SAW was switched on and off for a duration of $5 \mathrm{~s}$ at the power of $9 \mathrm{dBm}$. The vertical lines tag the time points when the SAW power is switched on/off.
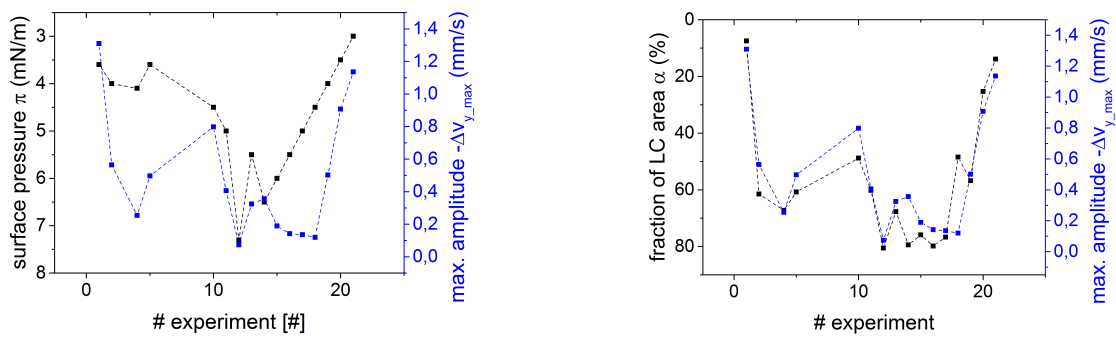

Fig. 11. a) A comparison of surface pressure and $\triangle v_{\mathrm{y}}$ shows only moderate correlation. However, the maximum velocity amplitude seems to lag behind the surface pressure. b) In contrast the graphs for the relative area of LC-domains and $v_{y \text {,max }}$ match very well. As a guide to the eye we connected the subsequent data points by lines to simplify the comparison in between the two graphs.

behavior is well known and has been reported in the literature [55]. The reason for this is the domain growth dynamics of these systems, which was intensively discussed [51], particularly the fact that labeled lipid monolayers may have to be regarded as a two-component system. If we choose the relative area of LC domains $\alpha$ instead of the surface pressure $\pi$ as the independent variable for further analysis, the correlation becomes much more significant, as can be seen in Figure 11b.

In Figure 12, we summarize the data obtained from a large number of experiments. A clear trend can be observed, even though there is quite a high scattering of the data points. The reasons for this become apparent when we consider the domain structure of the monolayer at different data points. As pointed out by Möhwald and others, the diffusion of dye molecules or other impurities controls the typical fractal domain growth $[49,50,33]$. Additional factors like history dependence and mechanical disturbances lead to the fact that the microstructure of lipid monolayers is not fully controlled in the transition regime. Therefore, it is not surprising that different domain 


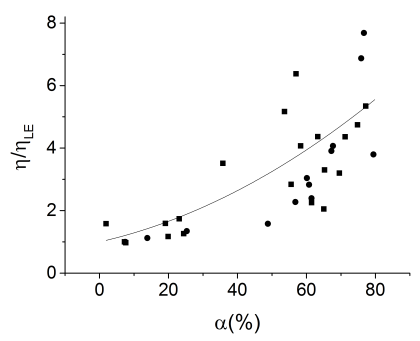

Fig. 12. Relative viscosity $\eta / \eta_{L E}$ as a function of the LC area proportion $\alpha$. The strong scattering of the measured values is most likely a consequence of a variation in domain microstructure. The continuous line is a fit using Einsteins equation with $C=4$ and indicates the increase of the viscosity ratio with $\alpha$.

structures lead to different shear properties. If we regard the monolayer in the transition regime as a system, which consists of more or less solid-like LCrafts floating in a fluid LE-matrix, it is obvious that its fine structure affects the flow. However, as a first attempt, we treat the LC-domains as weakly interacting circular discs and consider the system as a 2-dimensional quasicontinuous (emulsion-like) medium. The viscosity of such systems can usually be described empirically by a modified Einstein's equation [32, 22]

$$
\frac{\eta}{\eta_{0}}=1+2.5 \alpha+C \alpha^{2} .
$$

Here, $\eta_{0}$ is the viscosity of the fluid LE-phase. We further assume that $\triangle v_{\mathrm{y}} \propto F_{\mathrm{y}} / \eta \propto 1 / \eta$, where $F_{\mathrm{y}}$ denotes the $y$-component of the SAW streaming force field, which engages on the interface, we can fit our data with (4) after normalization with the maximum measured value for $\triangle v_{\mathrm{y}}$, which represents $1 / \eta$. For the solid line in Figure 12, we choose $C=4$. An empirical description of the system as a $2 \mathrm{D}$ colloid is thus feasible. For a more quantitative description, however, a precise analysis of the flow field as well as a refined model for the relation between surface viscosity and domain structure is necessary.

\section{Domain structure and viscoelasticity}

So far, the lipid monolayer was treated as purely viscous without considering elastic effects. However, a closer look at the data in Figure 10 reveals that there is an elastic contribution to the waveform of $\triangle v_{\mathrm{y}}$. This becomes much more pronounced for smaller SAW power, i.e. reduced excitation force (see Figure 13).

This elastic behavior can be observed in many experiments. However, the quantitative strength of it varies significantly between experiments and different ROIs in one experiment. Figure 14 shows a typical picture captured 


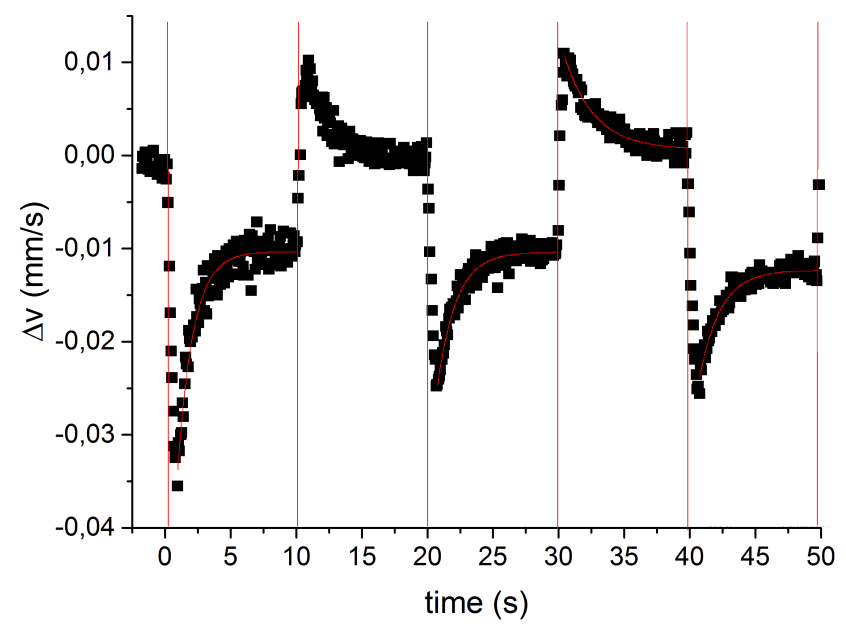

Fig. 13. $\triangle v_{\mathrm{y}}$ as a function of time for small SAW power. The reversal of the flow direction indicates a pronounced elastic contribution to the shear properties of the lipid monolayer.

during an experiment. There are two clearly distinguished regions of different domain morphology. Region one consists of isolated LC islands (disconnected), whereas region two exhibits a mesh-like domain structure (connected). Both morphologies were stable without mechanical disturbance and have similar LC proportion. However, the formation of morphology 2 was a result of a preceding SAW excitation and demonstrates that the domain structure can be influenced by slight disturbances. If one observes the dynamics of regions like the ones shown under flow excitation, one realizes that regions with unconnected domains behave more viscous, whereas those with connected domains show strong viscoelastic characteristics. One possibility for a quantification of the domain structure is the connectivity $\zeta$, which can be determined by the BoneJ plugin for ImageJ as shown in Figure 14. This clearly demonstrates that the shear viscosity of lipid monolayers in the LC-LE coexistence region depends strongly on the area proportion of LC domains as well as on the structure of the domains. A more thorough quantitative analysis of the dependence of the material properties on the connectivity is a very promising direction for further experimental and analytical studies of this highly interesting and complex system and remains the subject of ongoing work.

The development of a numerical model that considers the mutual influence of mechanical stress and domain structure is a first step towards a better understanding of the viscoelastic shear properties of lipid membranes in the vicinity of their phase transition. From a physical point of view, the sim- 

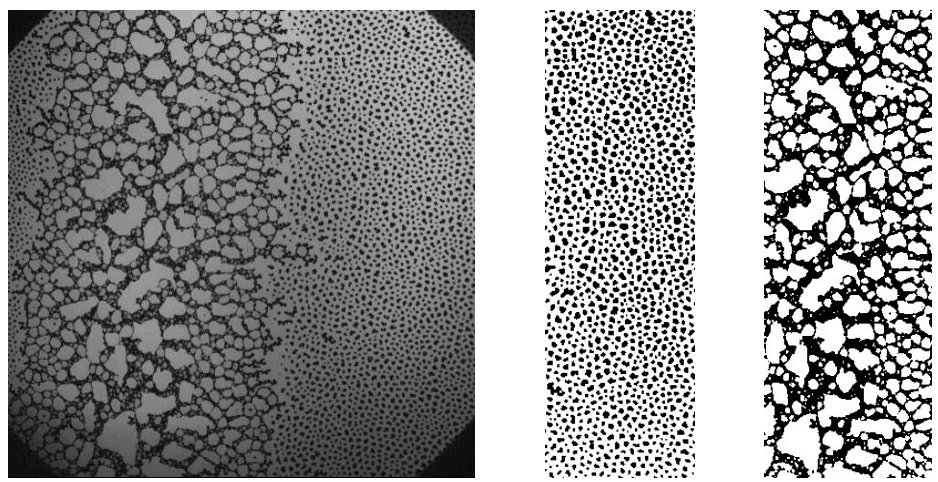

Fig. 14. Coexistence of two regions of very distinct morphology: a) original picture; b) "thresholded" picture region 1 (disconnected): $\zeta=-458$; c) "thresholded" picture region 2 (connected): $\zeta=+208$

plest mechanical model that can cover the observed dynamics is an extended Maxwell model as proposed by [45] for viscoelastic fluids. The combination of such a simple material model with a dynamic simulation of the quadrupolar force field for the SAW excitation is the subject of the following Section and can be a basis for later data analysis. A second step should be the identification of relations between the mechanical material parameters and the phase separation behavior of lipid membranes and a first attempt to develop such a model is presented in the next Section.

\section{Numerical simulation of the NS/CJ/CH system}

We consider a monolayer of lipids spread onto the upper surface of a waterfilled Langmuir-Blodgett trough and study phase separation under the influence of a surface-acoustic-wave-actuated fluid flow, which generates four counter-rotating vortices at the upper surface. The process can be described by the NS/CJ/CH system consisting of the incompressible Navier-Stokes equations with a viscoelastic stress term and a capillary term coupled with the convective Jeffreys (Oldroyd) equation of viscoelasticity and the Cahn-Hilliard equation with a transport term.

\subsection{The convective Jeffreys (Oldroyd) model of viscoelasticity}

Classical models of viscoelastic fluids are given by one-dimensional mechanical systems composed by springs and dashpots (cf., e.g., [67]). In particular, a Maxwell element consists of a spring and a dashpot in series. We denote by $\sigma$ the stress and by $\varepsilon$ the strain, and we refer to $G$ as the elastic modulus of the spring, to $\eta$ as the viscosity of the dashpot, and to $\tau_{\text {rel }}=\eta / G$ as the relaxation time. The constitutive equation for the Maxwell element reads 


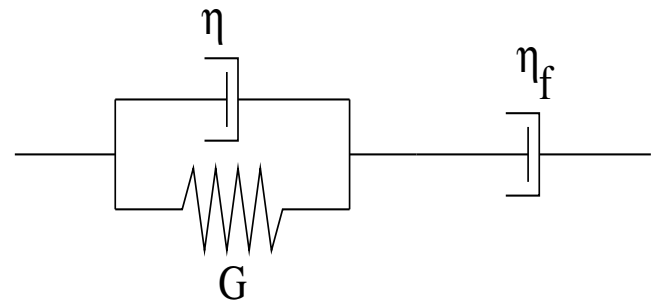

Fig. 15. The anti-Zener model also known as the non-standard three-parameter Voigt model or the Jeffreys model.

$$
\tau_{\text {rel }} \frac{\partial \sigma}{\partial t}+\sigma=\eta \frac{\partial \varepsilon}{\partial t}
$$

On the other hand, a Voigt element consists of a spring and a dashpot in parallel. Its constitutive equation is given by

$$
\sigma=G \varepsilon+\eta \frac{\partial \varepsilon}{\partial t}
$$

The Zener model [72] is a Voigt element in series with a spring and also referred to as the standard three-parameter Voigt model [67] with the constitutive equation

$$
a \frac{\partial \sigma}{\partial t}+\sigma=G \varepsilon+\eta \frac{\partial \varepsilon}{\partial t}
$$

where the additional parameter $a$ is related to the elastic modulus of the additional spring.

The anti-Zener model consists of a Voigt element in series with a dashpot of viscosity $\eta_{\mathrm{f}}$ (cf. Figure 15$)$. Denoting by $\tau_{\text {rel }}=\left(\eta+\eta_{\mathrm{f}}\right) / G$ the relaxation time and by $\tau_{\text {ret }}=\eta / G$ the retardation time, its constitutive equation is given by

$$
\tau_{\text {rel }} \frac{\partial \sigma}{\partial t}+\sigma=\eta_{\mathrm{f}}\left(\frac{\partial \varepsilon}{\partial t}+\tau_{\text {ret }} \frac{\partial^{2} \varepsilon}{\partial t^{2}}\right) .
$$

The anti-Zener model is also known as the non-standard three-parameter Voigt model [67] or the Jeffreys model [37].

We introduce the solvent (Newtonian) viscosity $\eta_{\mathrm{s}}$ and the elastic viscosity $\eta_{\mathrm{e}}$ according to

$$
\eta_{\mathrm{s}}=\eta_{\mathrm{f}} \frac{\tau_{\mathrm{ret}}}{\tau_{\text {rel }}}, \quad \eta_{\mathrm{e}}=\eta_{\mathrm{f}}\left(1-\frac{\tau_{\text {ret }}}{\tau_{\text {rel }}}\right) .
$$

Then, the stress $\sigma$ can be split into two parts by means of

$$
\sigma=\eta_{\mathrm{s}} \frac{\partial \varepsilon}{\partial t}+T
$$


The first part $\eta_{\mathrm{s}} \frac{\partial \varepsilon}{\partial t}$ is a Newtonian stress. The second part $T$ is the so-called extra stress, which satisfies

$$
\tau_{\text {rel }} \frac{\partial T}{\partial t}+T=\eta_{\mathrm{e}} \frac{\partial \varepsilon}{\partial t} .
$$

The explicit solution of (11) is given by

$$
T(t)=\exp \left(-t / \tau_{\text {rel }}\right) T(0)+\frac{\eta_{\mathrm{e}}}{\tau_{\text {rel }}} \int_{0}^{t} \exp \left(-(t-s) / \tau_{\text {rel }}\right) \frac{\partial \varepsilon}{\partial s}(s) \mathrm{d} s .
$$

Setting $T(0)=0$, we obtain

$$
\sigma(0)=\eta_{s} \frac{\partial \varepsilon}{\partial t}(0)
$$

i.e., at time $t=0$, the material experiences an immediate viscous response with the effective viscosity coefficient $\eta_{s}$. For the stress $\sigma$, we thus get

$$
\sigma(t)=\eta_{\mathrm{s}} \frac{\partial \varepsilon}{\partial t}(t)+\frac{\eta_{\mathrm{e}}}{\tau_{\text {rel }}} \int_{0}^{t} \exp \left(-(t-s) / \tau_{\text {rel }}\right) \frac{\partial \varepsilon}{\partial s}(s) \mathrm{d} s .
$$

By inverting (12) we obtain the dependence of the rate of strain on the history of the stress

$$
\frac{\partial \varepsilon}{\partial t}(t)=\frac{1}{\eta_{\mathrm{s}}} \sigma(t)-\frac{1}{\eta \tau_{\text {ret }}} \int_{0}^{t} \exp \left(-(t-s) / \tau_{\text {ret }}\right) \sigma(s) \mathrm{d} s .
$$

In terms of the displacement $u$, we have $\varepsilon=\partial u / \partial x$ for the strain and $v=$ $\partial u / \partial t$ for the velocity. It follows that $\partial \varepsilon / \partial t=\partial v / \partial x=\partial^{2} u /(\partial x \partial t)$. Hence, the anti-Zener (Jeffreys) model (8) can be written as

$$
\tau_{\text {rel }} \frac{\partial \sigma}{\partial t}+\sigma=\eta_{\mathrm{f}}\left(\frac{\partial v}{\partial x}+\tau_{\text {ret }} \frac{\partial}{\partial t}\left(\frac{\partial v}{\partial x}\right)\right) .
$$

This equation can be easily generalized to higher dimensions. We refer to $\mathbf{u}=\left(u_{1}, \cdots, u_{d}\right), d \in\{2,3\}$, as the displacement vector, to $\mathbf{v}=\partial \mathbf{u} / \partial t$ as the velocity, and to $\boldsymbol{\sigma}=\left(\sigma_{i j}\right)_{i, j=1}^{d}$ as the stress tensor. Further, let $\mathbf{D}(\mathbf{v})=$ $\left(\boldsymbol{\nabla} \mathbf{v}+\boldsymbol{\nabla} \mathbf{v}^{T}\right) / 2$ be the stretch tensor and $D / D t$ be any convective tensorial time derivative. Then, the $d$-dimensional anti-Zener (Jeffreys) model reads

$$
\tau_{\text {rel }} \frac{D \boldsymbol{\sigma}}{D t}+\boldsymbol{\sigma}=2 \eta_{\mathrm{f}}\left(\mathbf{D}(\mathbf{v})+\tau_{\text {ret }} \frac{D}{D t}(\mathbf{D}(\mathbf{v}))\right) .
$$

As a particular convective tensorial time derivative, we consider the oneparameter family of Gordon-Showalter derivatives [31]. For a tensor-valued function $\mathbf{G}$, this family is given by 


$$
\frac{D \mathbf{G}}{D t}=\frac{\partial \mathbf{G}}{\partial t}+\mathbf{v} \cdot \nabla \mathbf{G}-(a \mathbf{D}(\mathbf{v})+\mathbf{W}(\mathbf{v})) \mathbf{G}-\mathbf{G}(a \mathbf{D}(\mathbf{v})-\mathbf{W}(\mathbf{v})),
$$

where $\mathbf{W}(\mathbf{v})$ stands for the spin tensor $\mathbf{W}(\mathbf{v})=\left(\boldsymbol{\nabla} \mathbf{v}-\boldsymbol{\nabla} \mathbf{v}^{T}\right) / 2$. The parameter $a$, which defines the family, is supposed to lie in the interval $[-1,+1]$.

As in the one-dimensional case, the stress tensor $\sigma$ can be split into a Newtonian stress and an extra-stress according to

$$
\boldsymbol{\sigma}=2 \eta_{\mathbf{s}} \mathbf{D}(\mathbf{v})+\mathbf{T} .
$$

The extra-stress $\mathbf{T}$ satisfies the so-called Oldroyd viscoelastic equation [53]

$$
\tau_{\text {rel }} \frac{D \mathbf{T}}{D t}+\mathbf{T}=2 \eta_{\mathrm{e}} \mathbf{D}(\mathbf{v})
$$

The equation (15) with the Gordon-Showalter derivatives (16) is known as the convective Jeffreys or Oldroyd model. In particular, the choice $a=1$ results in the so-called Oldroyd-B fluid model.

For two-dimensional problems, the local existence of a weak solution and the global existence under the assumption of small data have been shown in [25]. For the parameter $a=0$ the global existence of a weak solution without the assumption of small data has been established in [47]. More recent global existence results can be found in [18] which, however, do not include the case $a=1$ (Oldroyd-B model).

\subsection{The NS/CJ/CH system}

We assume that the fluid volume in the Langmuir-Blodgett trough occupies a domain $(-\ell, \ell)^{2} \times(0, h)$, where $(-\ell, \ell)^{2}$ is the cross-section of the trough and $h$ is its height. We consider the viscoelastic fluid flow on the upper surface $(-\ell, \ell)^{2} \times\{h\}$ of the trough over the time interval $[0, T]$. As a model, we choose the NS/CJ/CH system which is a system of parabolic partial differential equations consisting of the incompressible Navier-Stokes equations for the velocity $\mathbf{v}$ and pressure $p$ coupled with a regularized convective Jeffreys (Oldroyd) model for the extra-stress $\boldsymbol{\sigma}$, and the Cahn-Hilliard equation for the order parameter $c$ (concentration of one of the components of the binary mixture). The system will be given in the dimensionless form. To this end, we introduce $L_{\text {ref }}, v_{\text {ref }}, \rho_{\text {ref }}$, and $\eta_{\text {ref }}$ as a reference length, velocity, density, and dynamic viscosity, respectively. The computational domain is $\Omega=\left(-\ell / L_{\mathrm{ref}}, \ell / L_{\mathrm{ref}}\right)^{2}$, which is $\Omega=(-1,1)^{2}$, if we choose $L_{\mathrm{ref}}=\ell$. We denote by $\mathbf{n}_{\Gamma}$ the exterior unit normal vector on the boundary $\Gamma=\partial \Omega$, and we set $Q=\Omega \times\left(0, T / T_{\text {ref }}\right)$ and $\Sigma=\Gamma \times\left(0, T / T_{\text {ref }}\right)$. We further denote by $T_{\text {ref }}=L_{\text {ref }} / v_{\text {ref }}$ the reference time. In dimensionless form, the NS/CJ/CH system represents an initial-boundary value problem given by the system of partial differential equations 


$$
\begin{array}{rc}
\rho\left(\frac{\partial \mathbf{v}}{\partial t}+\mathbf{v} \cdot \nabla \mathbf{v}\right)-\frac{1}{\operatorname{Re}} \eta_{\mathbf{s}} \Delta \mathbf{v}+\boldsymbol{\nabla} p-\boldsymbol{\nabla} \cdot \boldsymbol{\sigma}-\frac{1}{2} \mathrm{Ca} \mu(c) \boldsymbol{\nabla} c=\mathbf{f} & \text { in } Q \\
\boldsymbol{\nabla} \cdot \mathbf{v}=0 & \text { in } Q, \\
\frac{\partial \boldsymbol{\sigma}}{\partial t}+\mathbf{v} \cdot \boldsymbol{\nabla} \boldsymbol{\sigma}+\left(\frac{1}{\mathrm{Wi}} \mathbf{I}-\mathbf{M}(a)\right) \boldsymbol{\sigma}-\frac{2}{\mathrm{ReWi}} \eta_{\mathrm{e}} \mathbf{D}(\mathbf{v})-\kappa \Delta \boldsymbol{\sigma}=0 & \text { in } Q, \\
\mathbf{M}(a) \boldsymbol{\sigma}=(a \mathbf{D}(\mathbf{v})+\mathbf{W}(\mathbf{v})) \boldsymbol{\sigma}+\boldsymbol{\sigma}(a \mathbf{D}(\mathbf{v})-\mathbf{W}(\mathbf{v})), & \\
\frac{\partial c}{\partial t}+\mathbf{v} \cdot \boldsymbol{\nabla} c-\frac{1}{\mathrm{Pe}} \boldsymbol{\nabla} \cdot(M \boldsymbol{\nabla} \mu(c))=0 & \text { in } Q, \\
\mu(c)=F^{\prime}(c)-\varepsilon^{2} \Delta c &
\end{array}
$$

with the boundary conditions

$$
\mathbf{v}=\mathbf{0}, \quad \boldsymbol{\sigma} \mathbf{n}_{\Gamma}=\mathbf{0}, \quad \mathbf{n}_{\Gamma} \cdot \boldsymbol{\nabla} c=\mathbf{n}_{\Gamma} \cdot \boldsymbol{\nabla} \mu(c)=0 \quad \text { on } \Sigma,
$$

and the initial conditions

$$
\mathbf{v}(0)=\mathbf{0}, \quad \boldsymbol{\sigma}(0)=\mathbf{0}, \quad c(0)=c_{0} .
$$

Here, Ca, Pe, Re, and Wi refer to the capillary number, the Péclet number, the Reynolds number, and the Weissenberg number as given by

$$
\begin{aligned}
& \mathrm{Pe}=\frac{v_{\text {ref }}}{v_{\text {rel }}}, \quad \mathrm{Re}=\frac{\rho_{\text {ref }} v_{\text {ref }} L_{\text {ref }}}{\eta_{\text {ref }}}, \\
& \mathrm{Ca}=\frac{\eta_{\text {ref }} v_{\text {ref }}}{\gamma}, \mathrm{Wi}=\frac{\tau_{\text {rel }}}{T_{\text {ref }}},
\end{aligned}
$$

where $\gamma$ is the surface tension between the two phases, $v_{\text {rel }}$ is the characteristic relative velocity between the two phases, and $\tau_{\text {rel }}$ stands for the relaxation time of the convective Jeffreys model. The surface-acoustic-wave-actuated fluid flow can be modeled by the source term $\mathbf{f}$ in the incompressible Navier-Stokes equations

$$
\mathbf{f}=\left\{\begin{array}{ll}
\mathbf{f}_{\mathrm{q}}, & \text { if IDT is turned on } \\
\mathbf{0}, & \text { if IDT is turned off, }
\end{array} .\right.
$$

Here, $\mathbf{f}_{\mathrm{q}}$ represents a quadrupolar force field which can be motivated as follows: The velocity field of an incompressible two-dimensional fluid can be described by means of a scalar function $\Psi_{\mathrm{q}}\left(x_{1}, x_{2}\right)$ such that the velocity field follows as

$$
\mathbf{v}_{\mathrm{q}}\left(x_{1}, x_{2}\right)=\operatorname{curl}_{2 \mathrm{D}} \Psi_{\mathrm{q}}\left(x_{1}, x_{2}\right):=\left(\frac{\partial \Psi_{q}}{\partial x_{2}},-\frac{\partial \Psi_{\mathrm{q}}}{\partial x_{1}}\right)^{T} .
$$

It is the divergence free solution of the Stokes equation for a fluid driven by the force density

$$
\mathbf{f}_{\mathrm{q}}=-\eta_{\mathrm{w}} \Delta \mathbf{v}_{\mathrm{q}}
$$


where $\eta_{\mathrm{w}}$ is the viscosity of water. For the surface-acoustic-wave actuated flow field in $\Omega$ we use the quadrupolar stream function

$$
\Psi_{\mathrm{q}}\left(x_{1}, x_{2}\right)=v_{\mathrm{m}} \frac{\sqrt{3}}{\pi} \frac{\sin \left(\pi x_{1}\right) \sin \left(\pi x_{2}\right)}{\left(2-\cos \left(\pi x_{1}\right)\right)\left(2-\cos \left(\pi x_{2}\right)\right)},
$$

where $v_{\mathrm{m}}$ denotes the maximum value of the velocity magnitude depending on the frequency of the interdigital transducer that generates the surface acoustic waves. With this choice, the fluid flow exhibits a vorticity pattern consisting of four mutually counter-rotating vortices which is what is experimentally observed (cf. Figure 26 in subsection 5.4).

We further refer to $\rho$ as the mass density of the lipid, to $\mathbf{I}$ as the fourthorder identity tensor, to $\kappa>0$ as a regularization parameter in Oldroyd's equation, to $M$ as the mobility coefficient in the Cahn-Hilliard equation, to $F(c)=c^{4} / 4-c^{2} / 2$ as the coarse-grain free energy density function, to $c_{0}$ as a given initial concentration, and to $\varepsilon>0$ as a parameter related to the thickness of the diffuse interface between the two phases (also cf. Section 2.2 for a thorough discussion of the parameters of the Cahn-Hilliard model). Note that the coarse-grain energy density function was chosen differently from the one in Section 2.2 (c.f. [8]). Here, we assume that the pure phases correspond to the value of the order parameter $c= \pm 1$ (rather than $c=0$ and $c=1$ ).

\subsection{Numerical solution of the NS/CJ/CH system}

We solve the initial-boundary value problem (17a)-(17h) numerically by a splitting algorithm with respect to a partition of the time interval $[0, \bar{T}], \bar{T}=$ $T / T_{\text {ref }}$, into subintervals $\left[t_{n}, t_{n+1}\right]$ of length $\Delta t=\bar{T} / N, N \in \mathbb{N}, 0 \leq n \leq N-1$. We denote by $\mathbf{v}^{(n)}, p^{(n)}, \boldsymbol{\sigma}^{(n)}$, and $c^{(n)}$ approximations of $\mathbf{v}, p, \boldsymbol{\sigma}$, and $c$ at time $t=t_{n}$ and set the initial values $\mathbf{v}^{(0)}=\mathbf{0}, \boldsymbol{\sigma}^{(0)}=\mathbf{0}, c^{(0)}=c_{0}$.

\section{Step 1: Solution of the incompressible Navier-Stokes equations.}

Given $\mathbf{v}^{(n)}, \boldsymbol{\sigma}^{(n)}$, and $c^{(n)}$, we compute $\mathbf{v}^{(n+1)}$ by the Chorin-Temam projection method $[16,64,65]$. This involves the following three fractional steps:

\section{Step 1.1: Computation of a tentative velocity $\tilde{\mathbf{v}}^{(n+1)}$}

Compute $\tilde{\mathbf{v}}^{(n+1)}$ as the solution of the boundary value problem:

$$
\begin{aligned}
& \rho \frac{\tilde{\mathbf{v}}^{(n+1)}-\mathbf{v}^{(n)}}{\Delta t}+\rho \mathbf{v}^{(n)} \cdot \nabla \mathbf{v}^{(n)}-\frac{1}{\operatorname{Re}} \eta_{\mathrm{s}} \Delta \tilde{\mathbf{v}}^{(n+1)}- \\
& \boldsymbol{\nabla} \cdot \boldsymbol{\sigma}^{(n)}-\frac{1}{2} \operatorname{Ca} \mu\left(c^{(n)}\right) \boldsymbol{\nabla} c^{(n)}=\mathbf{f} \quad \text { in } \Omega, \\
& \tilde{\mathbf{v}}^{(n+1)}=\mathbf{0} \quad \text { on } \Gamma .
\end{aligned}
$$

\section{Step 1.2: Computation of the pressure $p^{(n+1)}$}


Compute $p^{(n+1)}$ as the solution of the Poisson problem:

$$
\begin{aligned}
& -\Delta p^{(n+1)}=-\frac{\rho}{\Delta t} \boldsymbol{\nabla} \cdot \tilde{\mathbf{v}}^{(n+1)} \quad \text { in } \Omega, \\
& \mathbf{n}_{\Gamma} \cdot \boldsymbol{\nabla} p^{(n+1)}=0 \quad \text { on } \Gamma \text {. }
\end{aligned}
$$

Step 1.3: Computation of the velocity $\mathbf{v}^{(n+1)}$

Compute $\mathbf{v}^{(n+1)}$ as the projection of the tentative velocity $\tilde{\mathbf{v}}^{(n+1)}$ onto the space of divergence-free functions:

$$
\begin{aligned}
\rho \frac{\mathbf{v}^{(n+1)}-\tilde{\mathbf{v}}^{(n+1)}}{\Delta t}+\nabla p^{(n+1)} & =\mathbf{0} & & \text { in } \Omega, \\
\mathbf{v}^{(n+1)} & =\mathbf{0} & & \text { on } \Gamma .
\end{aligned}
$$

\section{Step 2: Solution of the regularized convective Jeffreys model}

Compute $\boldsymbol{\sigma}^{(n+1)}$ by the following two fractional steps:

Step 2.1: Computation of a tentative stress $\tilde{\sigma}^{(n+1)}$

Compute $\tilde{\boldsymbol{\sigma}}^{(n+1)}$ as the solution of the regularized transport equation:

$$
\begin{aligned}
& \frac{\tilde{\boldsymbol{\sigma}}^{(n+1)}-\boldsymbol{\sigma}^{(n)}}{\Delta t}+\mathbf{v}^{(n+1)} \cdot \nabla \tilde{\boldsymbol{\sigma}}^{(n+1)}-\kappa \Delta \tilde{\boldsymbol{\sigma}}^{(n+1)}=\mathbf{0} \text { in } \Omega, \\
& \mathbf{n}_{\Gamma} \cdot \kappa \boldsymbol{\nabla} \tilde{\boldsymbol{\sigma}}^{(n+1)}=\mathbf{0} \quad \text { on } \Gamma \text {. }
\end{aligned}
$$

\section{Step 2.2: Computation of $\sigma^{(n+1)}$}

Compute $\boldsymbol{\sigma}^{(n+1)}$ as the solution of the implicitly-in-time discretized equation (17c) without the transport term:

$$
\begin{aligned}
& \frac{\boldsymbol{\sigma}^{(n+1)}-\tilde{\boldsymbol{\sigma}}^{(n+1)}}{\Delta t}+\left(\frac{1}{\mathrm{Wi}} \mathbf{I}-\mathbf{M}(a)\right) \boldsymbol{\sigma}^{(n+1)} \\
& -\kappa \Delta \boldsymbol{\sigma}^{(n+1)}-\frac{2}{\operatorname{ReWi}} \eta_{\mathrm{e}} \mathbf{D}\left(\mathbf{v}^{(n+1)}\right)=\mathbf{0} \quad \text { in } \Omega, \\
& \mathbf{n}_{\Gamma} \cdot \kappa \boldsymbol{\nabla} \boldsymbol{\sigma}^{(n+1)}=\mathbf{0} \text { on } \Gamma .
\end{aligned}
$$

\section{Step 3: Solution of the Cahn-Hilliard equation}

Compute $c^{(n+1)}$ as the solution of

$$
\begin{aligned}
\frac{c^{(n+1)}-c^{(n)}}{\Delta t}+\mathbf{v}^{(n+1)} \cdot \boldsymbol{\nabla} c^{(n+1)}-\frac{1}{\mathrm{Pe}} \boldsymbol{\nabla} \cdot\left(M \boldsymbol{\nabla} \mu\left(c^{(n+1)}\right)\right)=0 & \text { in } \Omega, \\
\mathbf{n}_{\Gamma} \cdot \boldsymbol{\nabla} c^{(n+1)}=\mathbf{n}_{\Gamma} \cdot \boldsymbol{\nabla} \mu\left(c^{(n+1)}\right)=0 & \text { on } \Gamma .
\end{aligned}
$$


The discretization in space is done by finite element approximations with respect to a uniform grid consisting of right isosceles triangles of mesh size $h=1 / 64$. In particular, the components of the velocities $\tilde{\mathbf{v}}^{(n)}$ and $\mathbf{v}^{(n)}$ as well as the components of the stress tensors $\tilde{\boldsymbol{\sigma}}^{(n)}$ and $\boldsymbol{\sigma}^{(n)}$ are approximated by $\mathrm{C}^{0}$-conforming Lagrangian finite elements of polynomial degree 2 , whereas the pressure $p^{(n)}$ is approximated by $\mathrm{C}^{0}$-conforming Lagrangian finite elements of polynomial degree 1 (cf., e.g., [19]). Finally, for the numerical solution of the implicit-in-time discretized Cahn-Hilliard equation we use a $\mathrm{C}^{0}$-InteriorPenalty Discontinuous-Galerkin method based on $\mathrm{C}^{0}$-conforming Lagrangian finite elements of polynomial degree 2 (cf. Section 2.2).

\subsection{Numerical results}

We have implemented the NS/CJ/CH system (17a)-(17h) for the parameters and the associated capillary, Péclet, Reynolds, and Weissenberg numbers given in Table 1 and Table 2. The algorithm described in the previous subsection has been applied with a time step size $\Delta t=1.0 \cdot 10^{-3}$ and a uniform finite element mesh of mesh size $h=1 / 64$.

Table 1. Parameters in the NS/CJ/CH system

\begin{tabular}{|c|c|c|c|c|c|c|}
\hline$\ell$ & $L_{\mathrm{ref}}$ & $T$ & $T_{\mathrm{ref}}$ & $T_{\mathrm{s}}$ & $\rho$ & $\rho_{\mathrm{w}}$ \\
\hline $1.0 \cdot 10^{-2}$ & $1.0 \cdot 10^{-2}$ & 65 & 1.0 & 10 & $1.0 \cdot 10^{3}$ & $1.0 \cdot 10^{3}$ \\
\hline \hline$\rho_{\mathrm{ref}}$ & $v_{\mathrm{m}}$ & $v_{\mathrm{ref}}$ & $v_{\mathrm{rel}}$ & $\eta_{\mathrm{w}}$ & $\eta_{\mathrm{s}, \mathrm{phase} 1}$ & $\eta_{e, \text { phase } 1}$ \\
\hline $1.0 \cdot 10^{3}$ & $1.0 \cdot 10^{-2}$ & $1.0 \cdot 10^{-2}$ & $1.0 \cdot 10^{-4}$ & $1.0 \cdot 10^{-3}$ & $7.5 \cdot 10^{-3}$ & $5.0 \cdot 10^{-3}$ \\
\hline \hline$\eta_{\mathrm{s}, \mathrm{phase} 2}$ & $\eta_{\mathrm{e}, \mathrm{phase} 2}$ & $\eta_{\mathrm{ref}}$ & $\tau_{\mathrm{rel}}$ & $\gamma$ & $\kappa$ & $a$ \\
\hline $7.5 \cdot 10^{-3}$ & $1.0 \cdot 10^{-3}$ & $1.0 \cdot 10^{-3}$ & 1.0 & $1.0 \cdot 10^{-6}$ & $1.0 \cdot 10^{-4}$ & 1.0 \\
\hline
\end{tabular}

In a first series of numerical simulations, we investigate the impact of viscoelasticity on the surface-acoustic-wave-generated velocity field at the upper surface of the Langmuir-Blodgett trough. We turn the interdigital transducer (IDT) on and off periodically with period $T_{\mathrm{s}}=10.0$. In particular, the IDT is 
Table 2. Capillary number, Péclet number, Reynolds number, and Weissenberg number

\begin{tabular}{|c|c|c|c|}
\hline $\mathrm{Ca}$ & $\mathrm{Pe}$ & $\mathrm{Re}$ & $\mathrm{Wi}$ \\
\hline $1.0 \cdot 10^{-2}$ & $1.0 \cdot 10^{2}$ & $1.0 \cdot 10^{2}$ & 1.0 \\
\hline
\end{tabular}
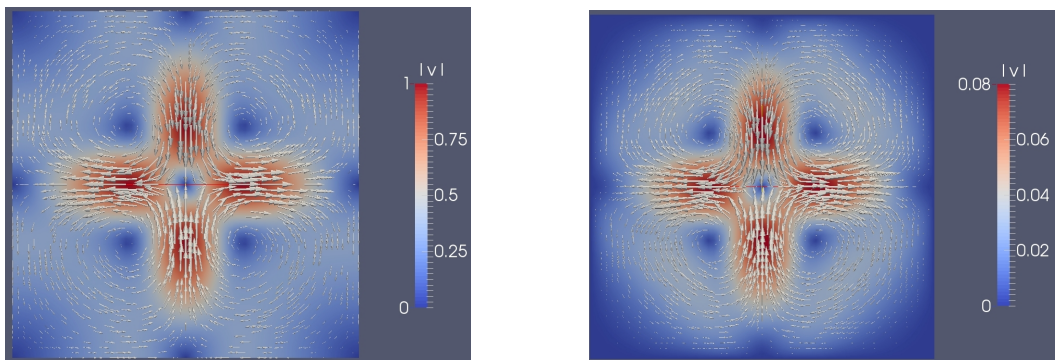

Fig. 16. Quadrupolar velocity field $\mathbf{v}_{\mathrm{q}}$ with $v_{\mathrm{m}}=1.0$ (left) and velocity $\mathbf{v}$ at time $t=10.00$ when the interdigital transducer is switched off (right).
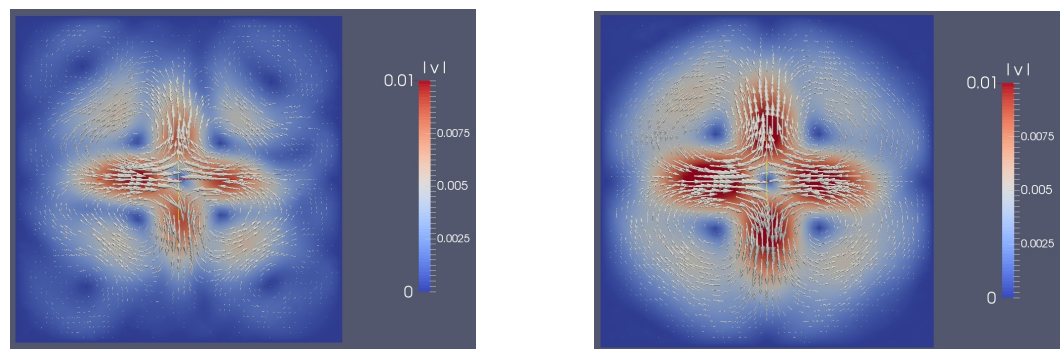

Fig. 17. Velocity field at time $t=11.25$ (left) and at time $t=11.50$ (right).

turned on at $t=0,2 T_{\mathrm{s}}, 4 T_{\mathrm{s}}, 6 T_{\mathrm{s}}$, and turned off at $t=T_{\mathrm{s}}, 3 T_{\mathrm{s}}, 5 T_{\mathrm{s}}$. Figure 16 displays the vorticity pattern consisting of four counter-rotating vortices corresponding to the quadrupolar velocity field $\mathbf{v}_{\mathrm{q}}$ with unit maximal magnitude $v_{\mathrm{m}}=1.0$ (left) and the computed velocity $\mathbf{v}$ at time $t=10.00$ (right) at the moment when the interdigital transducer is switched off. Figure 17 shows the velocity field at time $t=11.25$ (left) with the formation of counterflows in a vicinity of the origin and at time $t=11.50$ (right) with a complete change of the direction of the velocity and accelerated velocity magnitudes owing to the release of stored elastic energy.

The computed concentration profiles at different time moments are shown in Figure 18 and Figure 19. The left part of Figure 18 shows $c$ at $t=0.9$, 

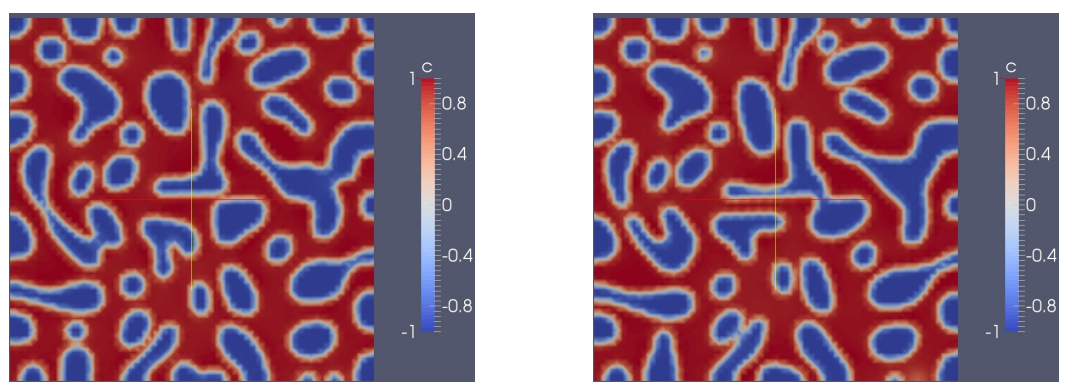

Fig. 18. Concentration at time $t=9.0$ (left), and at time $t=10.0$ (right).
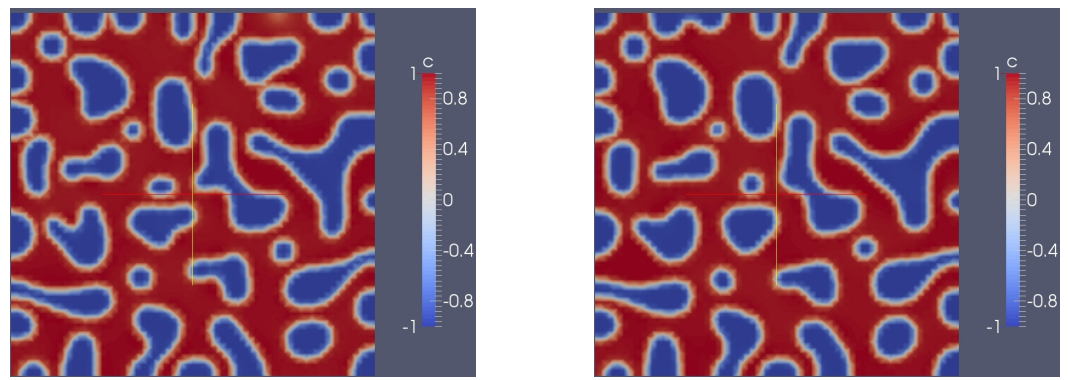

Fig. 19. Concentration at time $t=11.25$ (left), and at time $t=12.0$ (right).

while the right part corresponds to $t=10$, the exact time when the IDT is switched off. We can clearly see the movement of the pure phase domains in the direction of the quadrupolar force field. Figure 19 shows $c$ at $t=11.25$ (left) and $t=12.0$ (right). It can be seen that the direction of the flow is reversed for the short time.

We also choose a test point $\mathbf{x}_{0}=(2 / 32,14 / 32)$, which lies in the northeast quadrant near the line of separation of the counter-rotating vortices, and track the change in time of the vertical component $v_{\mathrm{y}}$ of the velocity $\mathbf{v}$ at this point. The results are shown on the Figure 20. We observe the typical viscoelastic flow pattern, namely,

- Initially the fluid is at rest (time $t=0$ );

- After switching the IDT on at $t=0, v_{\mathrm{y}}$ attains its maximal value (by modulus). In our case, this level is $\left|v_{\mathrm{y}}\right| \approx 0.08$;

- Then, it stabilizes at $\left|v_{\mathrm{y}}\right| \approx 0.07$. The wiggles indicate the effect of the heterogeneity of the domain as well as the elastic contribution;

- At time $t=10.0$ (corresponds to 1000 in the Figure), we turn the IDT off and observe the flow by inertia, i.e. $v_{\mathrm{y}}$ remains negative and $\left|v_{\mathrm{y}}\right|$ decreases;

- After becoming zero, the flow does not stop as it would in the viscous case. Rather, $v_{\mathrm{y}}$ reaches its maximum level $v_{\mathrm{y}} \approx 0.01$ (at this time we observe the maximum magnitude counter-flow). 


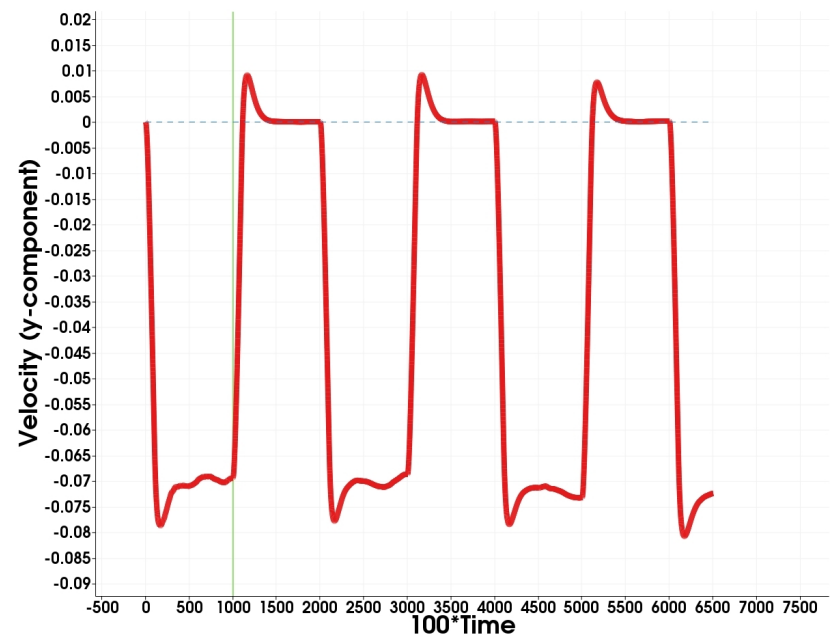

Fig. 20. Vertical component $v_{\mathrm{y}}$ of the velocity field $v$ at the test point $x_{0}=$ $(2 / 32,14 / 32)$.

- Then, the flow decays to zero. Due to elastic effects, it may cross zero back and forth several times with amplitude too small to be visible in the Figure.

Thus, qualitatively we observe the same effect as in the experiments, cf. Figure 13.

In the second series of experiments, we consider only a one-phase fluid. For numerical simulations, we use the system (17a)-(17c) with the value of the order parameter $c \equiv 1$ (therefore, the term $(1 / 2) \operatorname{Ca} \mu(c) \nabla c$ in the equation (17a) vanishes). Again, as in the two-phase case, the interdigital transducer is switched on at times $t=0,2 T_{\mathrm{s}}, 4 T_{\mathrm{s}}, 6 T_{\mathrm{s}}$ and switched off at times $t=T_{\mathrm{s}}, 3 T_{\mathrm{s}}, 5 T_{\mathrm{s}}$ with $T_{\mathrm{s}}=10.0$.

First, we simulate the flow of the pure viscous liquid $\left(\eta_{\mathrm{e}}=0\right)$ and the flow of the liquid-disordered phase of the lipid (phase 2 in the notations of Table 1$)$. The history of the $y$-component of the velocity $v_{\mathrm{y}}$ at the test point $(2 / 32,14 / 32)$ is shown in Figures 21 and 22 . We can see that $v_{\mathrm{y}}$ does not cross the zero level in the pure viscous case as expected whereas little elastic contribution to the model leads to small positive values which indicate very slow reverse motion.

Second, we simulate the flow of the condensed phase of the lipid (phase 1 in the notations of Table 1) and compare the simulation results with corresponding experimental measurements as shown in Figure 23. Qualitatively, the pictures look alike. Again, we see the crossing of zero of the vertical component $v_{\mathrm{y}}$ more expressed than for the liquid-disordered phase. In the experiments, the relaxation is more moderately expressed than in the case of simulations. A possible reason for this is a relatively low Weissenberg number $(\mathrm{Wi}=1.0)$ 


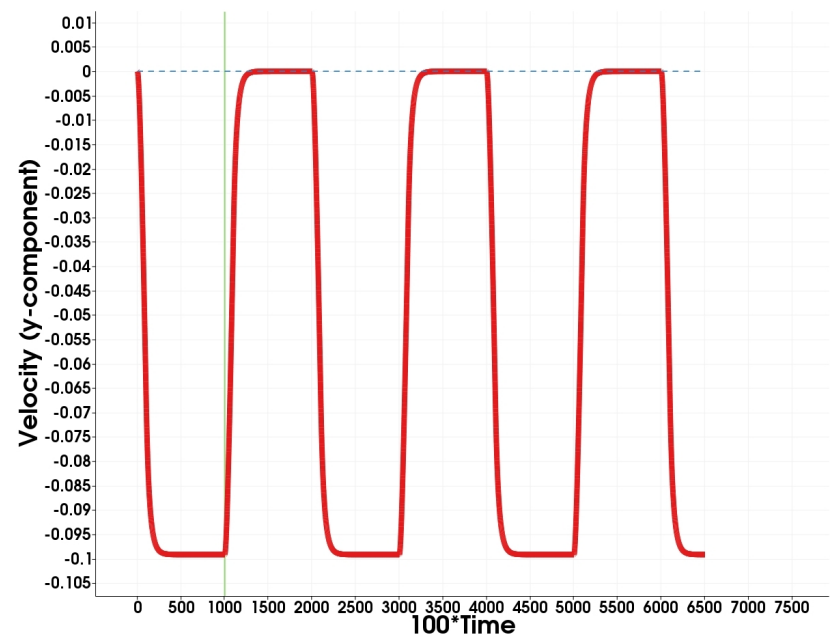

Fig. 21. Vertical component of the velocity at the test point. Simulation of the pure viscous liquid.

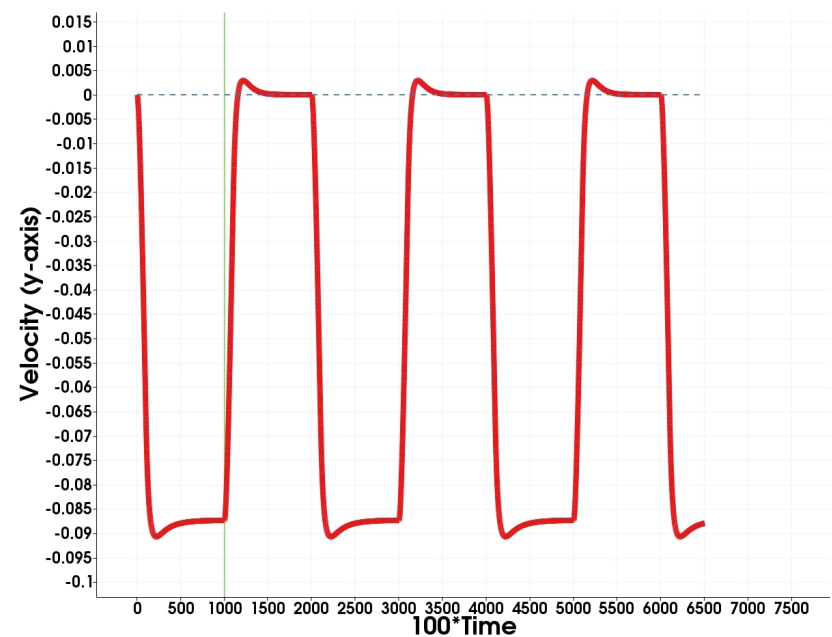

Fig. 22. Vertical component of the velocity at the test point. Simulation of the disordered phase of the lipid.

used. For larger values of Wi a more sophisticated numerical scheme to solve the convective Jeffreys equation would have to be implemented. 

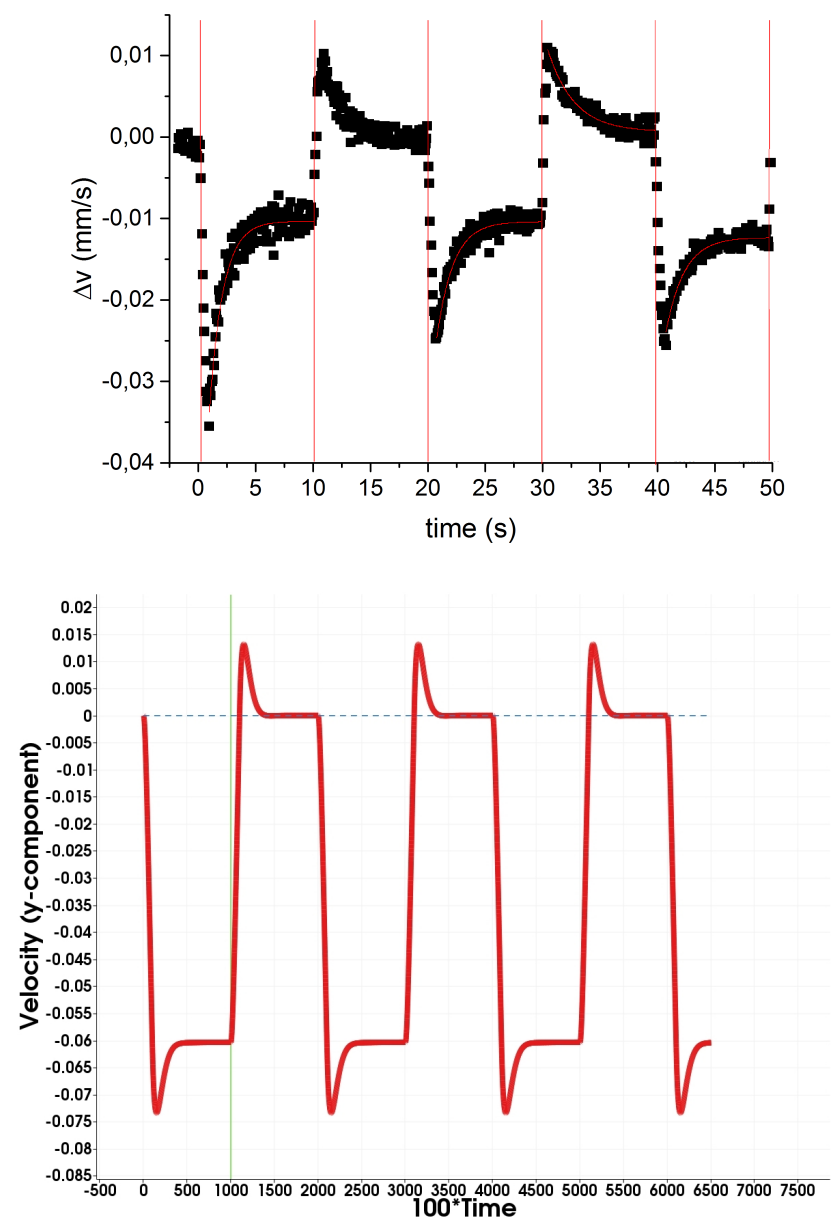

Fig. 23. Vertical component of the velocity at the test point. Top: experimental measurements. Bottom: simulation of the ordered (condensed) phase of the lipid. 

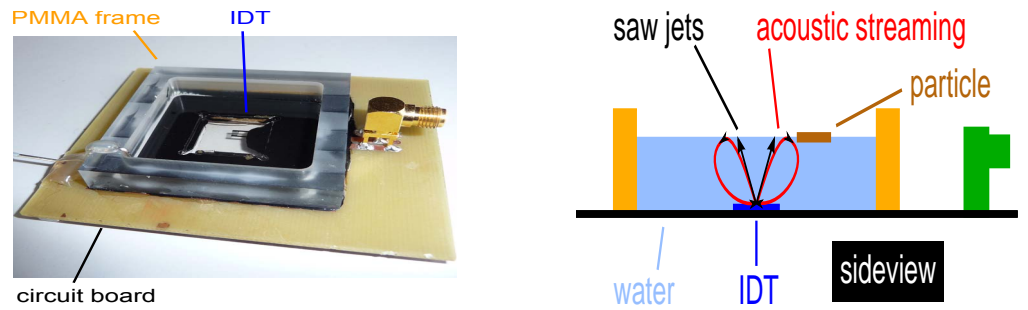

Fig. 24. Left: Photograph of the experimental setup. Right: Schematic sideview, where a particle is placed on the water surface and the IDT is driving the acoustic streaming.

\section{Enantiomer separation}

In this section, we report on the experimental setup for surface-acoustic-waveactuated enantiomer separation (subsection 5.1), the mathematical modeling and numerical simulation based on the finite element immersed boundary method (subsection 5.2) and the distributed Lagrangian multiplier finite element immersed boundary method (subsection 5.3), a comparison of experimental measurements and numerical simulation results (subsection 5.4), and the separation mechanism due to the specific flow pattern created by the surface acoustic waves (subsection 5.5).

\subsection{Experimental setup}

To drive the microfluidic flow, we apply surface acoustic waves (SAWs) that are generated by an interdigital transducer (IDT). An IDT consists of two interlocking electrodes deposited on a piezoelectric substrate, which are connected to an alternating current generator such that interfering periodic substrate deformations cause the propagation of SAWs. When SAWs couple into a liquid, two fluid jets are formed, which transport material. These jets drive a bulk streaming, which generates a specific flow pattern consisting of four counter-rotating vortices.

The experimental setup to apply SAW flow to the surface is shown in the photograph in Figure 24. An IDT is connected using a circuit board and a frame of plexiglas (PMMA) is attached to the board to form a miniaturized trough. The top right panel of the same figure shows a schematic sideview of the setup with the IDT on the bottom of the trough with a reservoir of water on top and a particle floating on the surface. Applying an alternating frequency drives the streaming and creates the specific flow pattern.

For the production of the photoresist L-shaped enantiomers, we followed the protocol described by [34] with some modifications. The whole process of manufacturing is shown in Figure 25 and the numbers in parenthesis in the following text also refer to that Figure. 


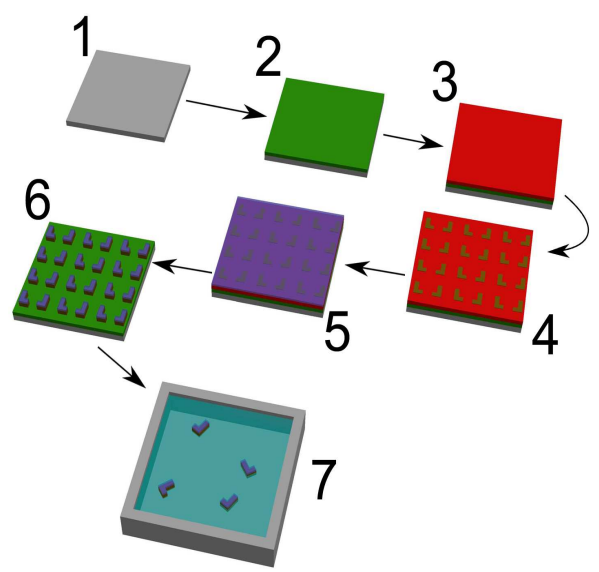

Fig. 25. Schematic representation of fabrication of the photoresist particles. 1) Clean silicon wafer. 2) A sacrificial layer of omnicoat is applied 3) SU8-2 is applied. 4) Photoresist is partially exposed. 5) Unexposed SU8-2 is developed. 6) Surface is treated with silane. 7) Lift-off. 8) Particles are brought onto water surface and float stably in well defined orientation. Reprinted with permission from S. Burger et al., Numerical simulation of surface acoustic wave actuated separation of rigid enantiomers by the fictitious domain Lagrange multiplier method. Comput. Meth. Appl. Math. 15 (3), 247-258. Copyright 2015 Walter de Gruyter.

First a sacrificial layer of omnicoat is spincoated on a silicon wafer (2). In a second spin coating process, SU8-2 photoresist laden with $0.5 \mathrm{mg} / \mathrm{ml}$ Nile Red is spun onto the omnicoat layer at 3000 rounds per second achieving a film thickness of about $1.5 \mu m$ (3). After soft baking, the photoresist is exposed using a mask aligner and then baked a second time (4). After the substrate has cooled to room temperature, the unexposed photoresist is developed using MR-DEV300 leaving the desired particles attached to the sacrificial layer of omnicoat (5). In order to render the particles hydrophobic on one side, a layer of Trichloro(octadecyl)silane (OTS) is applied by spin coating (6). To this end, $10 \mu \mathrm{l}$ OTS are dissolved in $3 \mathrm{ml} \mathrm{n}$-hexane and spun onto the particles at $1000 \mathrm{rpm}$ for 10 seconds. The OTS solution has to be applied after the spin coater has reached its maximum rate of revolution since n-hexane evaporates very quickly thus leaving the OTS scattered on the surface inhomogeneous if the spinning rate is too low. The layer of OTS renders the particles highly hydrophobic on the top side. After these steps, the particles are still firmly attached to the substrate and can be stored in a dark ambient until use to prevent bleaching of the fluorescent dye. To remove the particles from the wafer, a lift-off procedure is performed using omnicoat developer. The wafer is immersed in the solution until the omnicoat layer has been sufficiently dissolved, which takes approximately 30 seconds depending on the geometry of the sample. The wafer is then transferred to the experimental setup and 
the detached particles can be washed off using pure water. Due to the top side of the particles being considerably more hydrophobic than their bottom side, the orientation of the particles is conserved during the lift off process in most cases and the particles float stably on the surface of the fluid.

\subsection{Finite element immersed boundary method}

Following the experimental setup as described above, the simulation of the separation of L-shaped enantiomers has been considered by the Finite Element Immersed Boundary Method (FEIBM; cf. [7]). It is based on a coupled system consisting of the incompressible Navier-Stokes equations on the surface $\Omega$ of the fluid-filled container and the equations of motion of the enantiomers described with respect to an Eulerian and a Lagrangian coordinate system, respectively. Denoting by $\mathbf{u}, p$ the velocity and the pressure of the carrier fluid, by $\rho_{\mathrm{f}}, \eta_{\mathrm{f}}$ its density and viscosity, and by $\mathbf{X}$ the position vector on the boundary of length $\ell$ of an immersed enantiomer, the incompressible NavierStokes equations read

$$
\begin{aligned}
\rho_{\mathrm{f}}\left(\frac{\partial \mathbf{u}}{\partial t}+(\mathbf{u} \cdot \nabla) \mathbf{u}\right)-\eta_{\mathrm{f}} \Delta \mathbf{u}+\nabla p & =\mathbf{f}_{\mathrm{q}}+\mathbf{f}_{\mathrm{g}} \quad \text { in } \Omega \times(0, T), \\
\nabla \cdot \mathbf{u} & =0 \quad \text { in } \Omega \times(0, T), \\
\mathbf{u} & =\hat{\mathbf{u}} \quad \text { on } \partial \Omega \times(0, T), \\
\mathbf{u}(\cdot, 0) & =\hat{\mathbf{u}} \quad \text { in } \Omega,
\end{aligned}
$$

whereas the equation of motion is given by

$$
\begin{aligned}
\frac{d \mathbf{X}}{d t}(q, t) & =\mathbf{u}(\mathbf{X}(q, t), t), \quad q \in[0, \ell], t \in[0, T], \\
\mathbf{X}(q, 0) & =\mathbf{X}^{0}(q), \quad q \in[0, \ell],
\end{aligned}
$$

In (28a), the source term $\mathbf{f}_{\mathrm{q}}$ stands for the quadrupolar force density, cf. (19)$(21)$. On the other hand, the force density $\mathbf{f}_{\mathrm{g}}$ reflects the impact of the enantiomers on the carrier fluid according to

$$
\left\langle\mathbf{f}_{\mathrm{g}}(t), \mathbf{w}\right\rangle_{\mathbf{H}^{-1}, \mathbf{H}_{0}^{1}}=\int_{0}^{L} \mathbf{f}_{\mathbf{l}}(q, t) \cdot \mathbf{w}(\mathbf{X}(q, t)) \mathrm{d} q, \quad \mathbf{w} \in \mathbf{H}_{0}^{1}\left(\Gamma_{s}\right),
$$

where $\mathbf{H}_{0}^{1}$ is the vector-valued Sobolev space of square-integrable functions with square integrable weak derivative and vanishing boundary trace and $\mathbf{f}_{\mathbf{l}}(q, t)=-E^{\prime}(\mathbf{X}(q, t))$ with $E^{\prime}$ being the Gâteaux derivative of the total energy $E$ of the immersed body defined by

$$
\begin{aligned}
E(t) & :=E^{\mathrm{e}}(t)+E^{\mathrm{b}}(t), t \in(0, T), \\
E^{\mathrm{e}}(t) & :=\int_{0}^{L} \mathcal{E}^{\mathrm{e}}(\mathbf{X}(q, t)) \mathrm{d} q, \quad E^{\mathrm{b}}(t):=\int_{0}^{L} \mathcal{E}^{\mathrm{b}}(\mathbf{X}(q, t)) \mathrm{d} q .
\end{aligned}
$$


Here, $\mathcal{E}^{\mathrm{e}}(t)$ and $\mathcal{E}^{\mathrm{b}}(t)$ are the local energy densities according to

$$
\mathcal{E}^{\mathrm{e}}(\mathbf{X}(q, t))=\frac{\kappa_{e}}{2}\left(\left|\frac{\partial \mathbf{X}}{\partial q}(q, t)\right|^{2}-1\right), \quad \mathcal{E}^{\mathrm{b}}(\mathbf{X}(q, t))=\frac{\kappa_{b}}{2}\left|\frac{\partial^{2} \mathbf{X}}{\partial q^{2}}(q, t)\right|^{2}
$$

where $\kappa_{\mathrm{e}}>0$ and $\kappa_{\mathrm{b}}>0$ denote the elasticity coefficients with respect to elongation-compression and bending.

Based on the variational formulation of the coupled system (28),(29) in an appropriate function space setting, for the spatial discretization we have used Taylor-Hood P2/P1-elements for (28) with respect to a geometrically conforming simplicial triangulation $\mathcal{T}_{h}(\Omega)$ of the computational domain $\Omega$ and periodic cubic splines for (29) with respect to a partitioning of the interval $[0, L]$. Given an equidistant partition of the time interval $[0, T]$, for discretization in time we have used the implicit Euler scheme for (28) and the explicit Euler method for (29) resulting in a semi-implicit Backward Euler/Forward Euler FE-IB which has to satisfy a CFL-type stability condition (see also [27] for a related application of the FEIBM and [35] for an alternative unconditionally stable fully implicit scheme). For further details we refer to [5].

\subsection{Distributed Lagrangian multiplier finite element immersed boundary method}

In the Distributed Lagrangian Multiplier Finite Element Immersed Boundary Method (DLM-FEIBM) the rigid enantiomer is supposed to occupy a subdomain $B(t) \subset \Omega, t \in[0, T]$. The motion of the rigid enantiomer with density $\rho_{\mathrm{s}}$ and the first Piola-Kirchhoff stress tensor $\mathcal{P}$ is coupled by a distributed Lagrangian multiplier $\boldsymbol{\lambda} \in\left(H^{1}(B)^{2}\right)^{*}$. Hence, the DLM-FEIBM represents a fictitious domain approach in the spirit of [30]. In particular, setting $\mathbf{V}:=\left\{\mathbf{v} \in H^{1}(\Omega)^{2}|\mathbf{v}|_{\Gamma}=\hat{\mathbf{u}}\right\}$, the DLM-FEIBM requires the computation of $\mathbf{u} \in \mathbf{V}, p \in L_{0}^{2}(\Omega), \mathbf{X} \in H^{1}(B)^{2}$, and $\boldsymbol{\lambda} \in\left(H^{1}(B)^{2}\right)^{*}$ such that for all $\mathbf{v} \in H_{0}^{1}(\Omega)^{2}, q \in L_{0}^{2}(\Omega), \mathbf{Y} \in H^{1}(B)^{2}$, and $\boldsymbol{\mu} \in\left(H^{1}(B)^{2}\right)^{*}$ it holds

$$
\begin{aligned}
& \rho_{f} \int_{\Omega}\left(\frac{\partial \mathbf{u}}{\partial t}+(\mathbf{u} \cdot \nabla) \mathbf{u}\right) \cdot \mathbf{v} \mathrm{d} x+\eta_{\mathrm{f}} \int_{\Omega} \nabla \mathbf{u}: \nabla \mathbf{v} \mathrm{d} x \\
& -\int_{\Omega} p \nabla \cdot \mathbf{v} \mathrm{d} x+\langle\boldsymbol{\lambda}, \mathbf{v}(\mathbf{X}(\cdot, t))\rangle=\int_{\Omega} \mathbf{f}_{\mathrm{q}} \cdot \mathbf{v} \mathrm{d} x+\left\langle\mathbf{f}_{\mathrm{g}}, v\right\rangle_{\mathbf{H}^{-1}, \mathbf{H}_{0}^{1}}, \\
& \int_{\Omega} \nabla \cdot u q \mathrm{~d} x=0, \\
& \left(\rho_{\mathrm{s}}-\rho_{\mathrm{f}}\right) \int_{B} \frac{\partial^{2} \mathbf{X}}{\partial t^{2}} \cdot \mathbf{Y} \mathrm{d} x+\int_{B} \mathcal{P}: \nabla Y \mathrm{~d} x-\langle\lambda, \mathbf{Y}\rangle=0 \\
& \left\langle\boldsymbol{\mu}, \mathbf{u}(\mathbf{X}(\cdot, t))-\frac{\partial X}{\partial t}\right\rangle=0, \\
& \mathbf{u}(\cdot, 0)=\hat{\mathbf{u}} \text { in } \Omega, \quad \mathbf{X}(\cdot, 0)=\mathbf{X}^{0}
\end{aligned}
$$



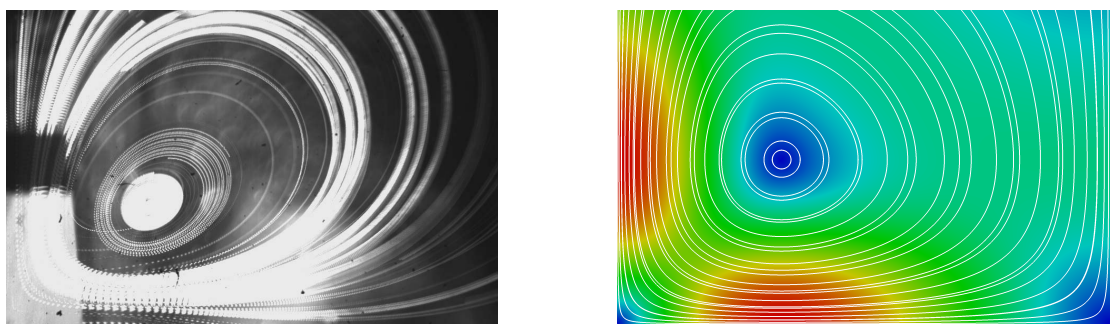

Fig. 26. Left: Experimental SAW generated surface streaming profile. Right: Simulated vorticity pattern generated by the quadrupolar force field. Reprinted with permission from S. Burger et al., Numerical simulation of surface acoustic wave actuated separation of rigid enantiomers by the fictitious domain Lagrange multiplier method. Comput. Meth. Appl. Math. 15 (3), 247-258. Copyright 2015 Walter de Gruyter.

where $\langle\cdot, \cdot\rangle$ stands for the duality pairing between $\left(H^{1}(B)^{2}\right)^{*}$ and $H^{1}(B)^{2}$.

For the numerical solution of the DLM-FEIBM we use inf-sup stable Taylor-Hood $P_{2} / P_{1}$-elements for the approximation of $(\mathbf{u}, p)$, conforming $P_{1^{-}}$ elements for the approximation of $\mathbf{X}$ and $\boldsymbol{\lambda}$, and the Yanenko-Marchuk fractional step method combined with a Chorin-Marsden splitting for discretization in time. For details we refer to [11].

\subsection{Comparison of experimental and simulation results}

The SAW-induced flow field at the surface and the motion of particles within that flow field can be easily visualized using either bright field or fluorescence microscopy. Figure 26 (left) displays the experimentally observed flow field in the upper-right quadrant of the surface generated by the SAWs, whereas Figure 26 (right) shows the simulated flow pattern generated by the quadrupolar force field.

Moreover, Figure 27 (left) shows a micrograph of the experiment and tracked trajectories of two particles (solid colored lines) as they pass through the center of the flow field. The streamlines are visualized by calculating an overlay of many frames. Figure 27 (right) displays the simulated trajectories based on the DLM-FEIBM.

\subsection{Numerical simulation of enantiomer separation}

As far as enantiomer separation is concerned, Figure 28 displays the motion of a right-handed and a left-handed L-shaped enantiomer initially placed between the two counter-rotating vortices in the lower quadrants of the surface of the fluid as simulated using the DLM-FEIBM. As can be seen in Figure 28 (left), the right-handed enantiomer gets attracted by the counter-clockwise rotating vortex in the lower-left quadrant. On the other hand, as shown in 

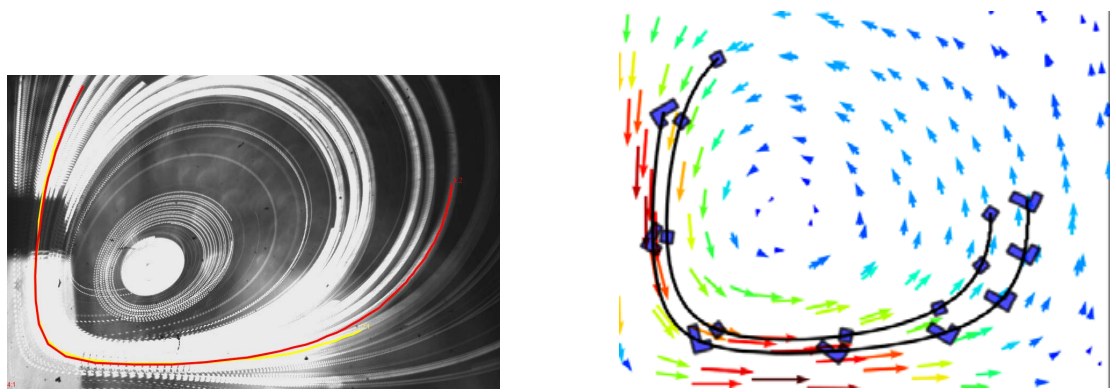

Fig. 27. Left: Experimentally measured trajectories of a square shaped particle (red line) and an L-shaped particle (yellow line). Right: Simulated trajectories. Reprinted with permission from S. Burger et al., Numerical simulation of surface acoustic wave actuated separation of rigid enantiomers by the fictitious domain Lagrange multiplier method. Comput. Meth. Appl. Math. 15 (3), 247-258. Copyright 2015 Walter de Gruyter.

Figure 28 (right), the right-handed enantiomer follows a trajectory that leads to a path around the center of the clockwise rotating vortex in the upper-right quadrant. Likewise, Figure 29 shows that a right-handed (left-handed) enantiomer initially placed a little bit to the right of the middle between the two counter-rotating vortices gets attracted by the counter-clockwise (clockwise) rotating vortex in the upper-right (lower-right) quadrant. This behavior is in accordance with experimental measurements and similar to numerical simulation results that have been obtained for deformable L-shaped enantiomers by an application of the FEIBM [5]. We note that [5] also contains numerical results for multiple enantiomers of different handedness which confirm the separation mechanism described above.

\section{Acknowledgements}

This work was supported by the DFG Priority Program SPP 1506, by the German Cluster of Excellence "Nanosystems Initiative Munich (NIM)", and by the NSF under grant DMS-1520886.

\section{References}

1. M. Angelova, S. Soléau, Ph. Méléard, F. Faucon, and P. Bothorel, Preparation of Giant Vesicles by external AC electric fields. Kinetics and applications. In: Trends in Colloid and Interface Science vol VI (eds. C. Helm, M. Lösche, and H. Möhwald). Series: Progress in Colloid and Polymer Science vol. 89. Springer Berlin-Heidelberg, 127-131, 1992. 

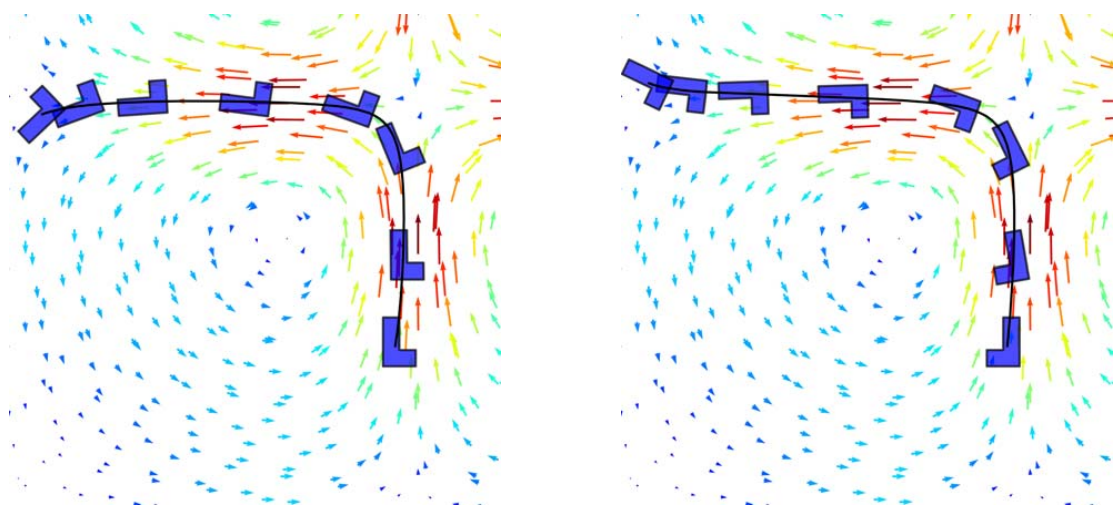

Fig. 28. Left: Attraction of a right-handed L-shaped enantiomer by the counterclockwise rotating vortex in the lower-left quadrant. Right: Attraction of a lefthanded L-shaped enantiomer by the clockwise rotating vortex in the upper-left quadrant. Reprinted with permission from S. Burger et al., Numerical simulation of surface acoustic wave actuated separation of rigid enantiomers by the fictitious domain Lagrange multiplier method. Comput. Meth. Appl. Math. 15 (3), 247-258. Copyright 2015 Walter de Gruyter.
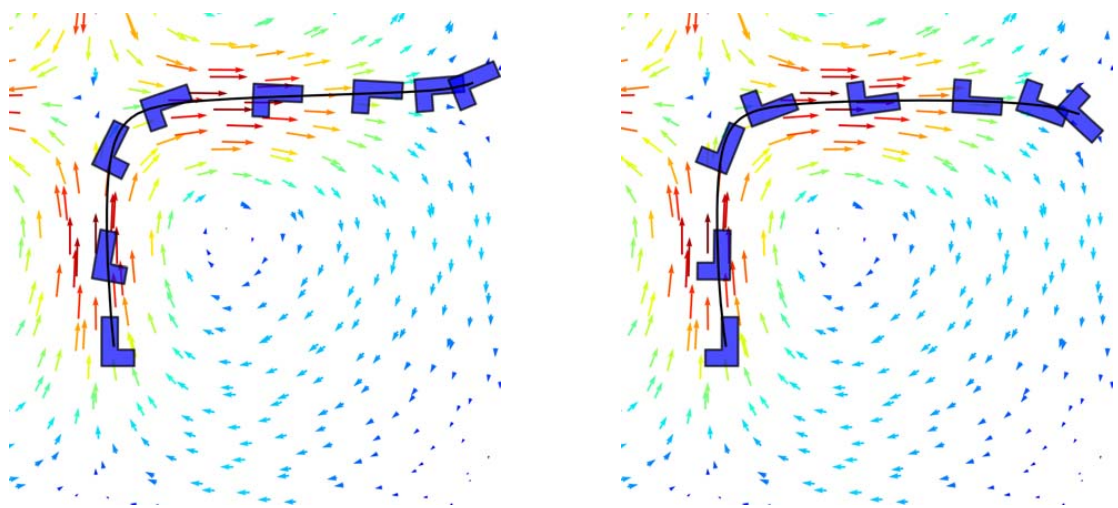

Fig. 29. Left: Attraction of a right-handed L-shaped enantiomer by the counterclockwise rotating vortex in the upper-right quadrant. Right: Attraction of a lefthanded L-shaped enantiomer by the clockwise rotating vortex in the lower-right quadrant.

2. L.A. Bagatolli and E. Gratton, Two photon fluorescence microscopy of coexisting lipid domains in Giant Unilamellar Vesicles of binary phospholipid mixtures. Biophysical Journal 78, 290-305, 2000.

3. S. Baoukina, E. Mendez-Villuendas, W. Bennett, and D. Tieleman, Computer simulations of the phase separation in model membranes. Faraday Discuss. 161, 63-75, 2013.

4. T. Baumgart, S. Hess, and W. Webb, Imaging coexisting fluid domains in biomembrane models coupling curvature and line tension. Nature 425, 821- 
824, 2003.

5. K. Beleke-Maxwell, T. Franke, R.H.W. Hoppe, and C. Linsenmann, Numerical simulation of surface acoustic wave actuated enantiomer separation by the finite element immersed boundary method. Computers and Fluids 112, 50-60, 2015.

6. A. Bertozzi, S. Esedoglu, and A. Gillette, Inpainting of binary images using the Cahn-Hilliard equation. IEEE Trans. Image Process. 16, 285-291, 2007.

7. D. Boffi and L. Gastaldi, A finite element approach for the immersed boundary method. Comput. Struct. 81, 491-501, 2003.

8. F. Boyer, A theoretical and numerical model for the study of incompressible mixture flows. Comput. Fluids 31, 41-68, 2002.

9. F. Boyer, L. Chupin, and P. Fabrie, Numerical study of viscoelastic mixtures through a Cahn-Hilliard flow model. Europ. J. of Mechanics B/Fluids 23, 759 780,2004

10. C.F. Brooks, G.G. Fuller, C.W. Frank, and C.R. Robertson, Transitions in monolayers at the air-water interface. Langmuir 5, 2450-2459, 1999.

11. S. Burger, T. Franke, T. Fraunholz, R.H.W. Hoppe, M.A. Peter, and A. Wixforth, Numerical simulation of surface acoustic wave actuated separation of rigid enantiomers by the fictitious domain Lagrange multiplier method. Comput. Meth. Appl. Math. 15, 247-258, 2015.

12. S. Burger, T. Fraunholz, R.H.W. Hoppe, C. Leirer, A. Wixforth, M.A. Peter, and T. Franke, Comparative study of the dynamics of lipid membrane phase decomposition in experiment and simulation. Langmuir 29, 7565-7570, 2013.

13. J.W. Cahn and J.E. Hilliard, Free energy of a non-uniform system. I. Interfacial free energy. J. Chem. Phys. 28, 258-267, 1958.

14. L. Chen, Phase-field models for microstructural evolution. Ann. Rev. Mater. Res. 32, 113-140, 2002.

15. S.Q. Choi, S. Steltenkamp, J.A. Zasadzinski, T.M. Squires, Active microrheology and simultaneous visualization of sheared phospholipid monolayers. Nature Communications 2, 312-316, 2011.

16. A.J. Chorin, The numerical solution of the Navier-Stokes equations for an incompressible fluid. Bull. Am. Soc. 73, 928-931, 1967.

17. L. Chupin, Existence result for a mixture of non-Newtonian flows with stress diffusion using the Cahn-Hilliard formulation. Discrete and Continuous Dynamical Systems B 3, 45-68, 2003.

18. L. Chupin, Existence results for the flow of viscoelastic fluids with an integral constitutive law. J. Math. Fluid Mech. 15, 783-806, 2013.

19. P.G. Ciarlet, The Finite Element Method for Elliptic Problems. SIAM, Philadelphia, 2002.

20. C. Dietrich, L. Bagatolli, Z. Volovyk, N. Thompson, M. Levi, K. Jacobson, and E. Gratton, Lipid rafts reconstituted in model membranes. Biophys. J. 80, 1417-1428, 2001.

21. J. Ding, H. E. Warriner, J.A. Zasadzinski, and D.K. Schwartz, Magnetic needle viscometer for Langmuir monolayers. Langmuir 18, 2800-2806, 2002.

22. A. Einstein, Eine neue Bestimmung der Moleküldimensionen. Annalen der Physik 324, 289-306, 1906.

23. C. Elliott, The Cahn-Hilliard model for the kinetics of phase separation. In: Mathematical models for phase change problems (ed. J. Rodrigues), International Series of Numerical Mathematics, Vol. 88. Birkhauser Publishing, Basel, pp. 35-74, 1989. 
24. G. Espinosa, I. López-Montero, F. Monroy, and D. Langevin, Shear rheology of lipid monolayers and insights on membrane fluidity. Proc. Nat. Ac. Sci. 108, 6008-6013, 2011.

25. E. Fernandéz-Cara, F. Guillén, and R.R. Ortega, Some theoretical results concerning non-Newtonian fluids of the Oldroyd kind. Ann. Scuola Norm. Sup. Pisa Cl. Sci. 26, 1-26, 1998.

26. A. Filippov, G. Orädd, and G. Lindblom. Lipid lateral diffusion in ordered and disordered phases in raft mixtures. Biophys. J. 86, 891-896, 2004.

27. T. Franke, R. H. W. Hoppe, C. Linsenmann, L. Schmidt, and C. Willbold, Numerical simulation of the motion of red blood cells and vesicles in microfluidic flows. Computing and Visualization in Science 14, 167-180, 2011.

28. T. Fraunholz, R.H.W. Hoppe, and M.A. Peter, Convergence analysis of an adaptive interior penalty discontinuous Galerkin method for the biharmonic problem. J. Numer. Math. 23, 317-330, 2015.

29. T. Fraunholz, Transport at Interfaces in Lipid Membranes and Enantiomer Separation. PhD dissertation, University of Augsburg, Germany, 2014.

30. R. Glowinski, T.-W. Pan, T.I. Hesla, D.D. Joseph, and J. Periaux, A fictitious domain approach to the direct numerical simulation of incompressible viscous flow past moving rigid bodies: Application to particulate flow. J. Comput. Phys. 169, 363-427, 2001.

31. R.J. Gordon and W.R. Showalter, Anisotropic fluid theory: a different approach to the dumbbell theory of dilute polymer solutions. Trans. Soc. Rheol. 16, 7997, 1972 .

32. E. Guth and R. Simha, Untersuchungen über die Viskosität von Suspensionen und Lösungen. 3. Über die Viskosität von Kugelsuspensionen. Colloid \& Polymer Science 74, 266-275, 1936.

33. C. Helm, H. Möhwald, K. Kjaer, and J. Als-Nielsen, Phospholipid monolayers between fluid and solid states. Biophysical journal 52, 381-390, 1987.

34. C.J. Hernandez and T.G. Mason, Colloidal Alphabet Soup: Monodisperse Dispersions of Shape-Designed LithoParticles. The Journal of Physical Chemistry C 111, 4477-4480, 2007.

35. R.H.W. Hoppe and C. Linsenmann, An adaptive Newton continuation strategy for the fully implicit finite element immersed boundary method. J. Comp. Phys. 231, 4676-4693, 2012.

36. K. Jacobson, O. Mouritsen, and R. Anderson, Lipid rafts: at a crossroad between cell biology and physics. Nature Cell Biology 9, 7-14, 2007.

37. H. Jeffreys, The Earth, its Origin, History and Physical Constitution. Cambridge University Press, 1924.

38. K. Jørgensen and O. Mouritsen, Phase separation dynamics and lateral organization of two-component lipid membranes. Biophys. J. 95, 942-954, 1995.

39. D.D. Joseph, Fluid Dynamics of Viscoelastic Liquids. Springer, BerlinHeidelberg-New York, 1990.

40. N. Kahya, D. Scherfeld, K. Bacia, B. Poolman, and P. Schwille, Probing lipid mobility of raft-exhibiting model membranes by fluorescence correlation spectroscopy. J. Biol. Chem. 278, 28109-28115, 2003.

41. J. Krägel, G. Kretzschmar, J.B. Li, G. Loglio, R. Miller, and H. Möhwald, Surface rheology of monolayers. Thin Solid Films 284-285, 361-364, 1996.

42. P. Krüger and M. Lösche, Molecular chirality and domain shapes in lipid monolayers on aqueous surfaces. Phys. Rev. E 62, 7031-7043, 2000. 
43. M. Kostur, M. Schindler, P. Talkner, and P. Hänggi, Chiral separation in microflows. Phys. Rev. Lett. 96, 014502-1 - 014502-4, 2006.

44. R.G. Larson, The Structure and Rheology of Complex Fluids. Oxford University Press, Oxford, 1999.

45. W. Letherish, The mechanical behavior of Bitumen. Journal of the Society of Chemical Industry 59, 1-26, 1940.

46. P.C.H. Li, Microfluidic Lab-on-a-Chip for Chemical and Biological Analysis and Discovery. CRC Press, Boca Raton, 2006.

47. P.L. Lions and N. Masmoudi, Global solutions for some Oldroyd models of non-Newtonian fluids. Chinese Ann. Math. Ser. B 21, 131-146, 2000.

48. Marcos, H.C. Fu, T.R. Powers, and R. Stocker, Separation of microscale chiral objects by shear flow. Phys. Rev. Lett. 102, 158103-1 - 158103-4, 2009.

49. A. Miller, W. Knol, and H. Möhwald, Fractal growth of crystalline phospholipid domains in monomolecular layers. Phys. Rev. Lett. 56, 2633-2638, 1986.

50. A. Miller and H. Möhwald, Diffusion limited growth of crystalline domains in phospholipid monolayers. J. Chem. Phys. 86, 4258i-4265, 1987.

51. H. Möhwald, Phospholipid Monolayers. In: Handbook of Biological Physics vol. 1, eds. R. Lipowsky and E. Sackmann, Elsevier Science B.V., 161-211, 1995.

52. A. Novick-Cohen, The Cahn-Hilliard equation: mathematical and modeling perspectives. Adv. Math. Sci. Appl. 8, 965-985, 1998.

53. J.G. Oldroyd, On the formulation of rheological equations of state. Proc. Roy. Soc. A 200, 523-541, 1950.

54. G. Orädd, P. Westerman, and G. Lindblom. Lateral diffusion coefficients of separate lipid species in a ternary raft-forming bilayer: A Pfg-NMR multinuclear study. Biophys. J. 89, 315-320, 2005.

55. R. Pichot, R.L. Watson, and I.T. Norton, Phospholipids at the interface: current trends and challenges. International Journal of Molecular Sciences 14, 1176711794, 2013.

56. A. Relini, F. Ciuchi, and R. Rolandi, Surface shear viscosity and phase transitions of monolayers at the air-water interface. Journal de Physique II 5, 12091221, 1995.

57. J. Rowlinson, and B. Widom, Molecular Theory of Capillarity. Clarendon Press, Oxford, U.K., 1982.

58. M. Sacchetti, H. Yu, G. Zografi, In-plane steady shear viscosity of monolayers at the air/water interface and its dependence on free area. Langmuir 9, 2168-2171, 1993.

59. A.H. Sadoughi, J.M. Lopez, and A.H. Hirsa, Transition from Newtonian to nonNewtonian surface shear viscosity of phospholipid monolayers. Phys. Fluids 25, 032107, 2013.

60. M. Sickert and F. Rondelez, Shear viscosity of Langmuir monolayers in the low-density limit. Phys. Rev. Lett. 90, 126104, 2003.

61. K. Simons and E. Ikonen, Functional rafts in cell membranes. Nature 387, 569-572, 1997.

62. S.J. Singer and J.L. Nicolson, The fluid mosaic model of the structure of cell membranes. Science 175, 720-735, 1972.

63. D. Steppich, J. Griesbauer, T. Frommelt, W. Appelt, A. Wixforth, and M.F. Schneider, Thermomechanic-electrical coupling in phospholipid monolayers near the critical point. Phys. Rev. E, 81, 061123, 2010. 
64. R. Temam, Remark on the pressure boundary condition for the projection method. Theoretical and Computational Fluid Mechanics 3, 181-184, 1991.

65. R. Temam, Navier-Stokes Equations: Theory and Numerical Analysis. AMS Chelsea Publications, Providence, Rhode Island, 2000.

66. S. Tremaine. On the origin of irregular structure in Saturn's rings. Astron. J. 125, 894-901, 2003.

67. N.W. Tschoegl, The Phenomenological Theory of Linear Viscoelastic Behavior. Springer, Berlin-Heidelberg-New York, 1989.

68. S. Veatch, and S. Keller, Organization in lipid membranes containing cholesterol. Phys. Rev. Lett. 89, 268101, 2002.

69. S. Veatch, and S. Keller, Separation of liquid phases in giant vesicles of ternary mixtures of phospholipids and cholesterol. Biophysical Journal 85, 3074-3083, 2003.

70. S. Veatch, and S. Keller, Seeing spots: complex phase behavior in simple membranes. Biochim. Biophys. Acta 1746, 172-185, 2005.

71. G.N. Wells, E. Kuhl, and K. Garikipati, A discontinuous Galerkin method for the Cahn-Hilliard equation. J. Comp. Phys. 218, 860-877, 2006.

72. C. Zener, Elasticity and Anelasticity of Metals. University of Chicago Press, Chicago, 1948. 